\title{
The Role of Asset Prices in Forecasting Inflation and Output in South Africa ${ }^{\#}$
}

\author{
Rangan Gupta* and Faaiqa Hartley**
}

\begin{abstract}
This paper assesses the predictive ability of asset prices relative to other variables in forecasting inflation and real GDP growth in South Africa. A total of 42 asset and non-asset predictor variables are considered. Forecasts of inflation and real GDP growth are computed using both individual predictor autoregressive distributed lag (ARDL) models, forecast combination approaches, as well as, large scale models. The large scale data models considered include Bayesian vector autoregressive models, and classical and Bayesian univariate and multivariate factor augmented vector autoregressive models. The models are estimated for an in-sample of 1980:Q2 to 1999:Q4, and then one- to eight step-ahead forecasts for inflation and real GDP growth are evaluated over the 2000:Q1 to 2010:Q2 out-of-sample period. Principle Component forecast combination models are found to produce the most accurate out-of-sample forecasts of inflation and real GDP growth relative to the other combination and more sophisticated models considered. Asset prices are found to contain particularly useful information for forecasting inflation and real GDP growth at certain horizons. Asset prices are however found to be stronger predictors of inflation, particularly in the long run.
\end{abstract}

Keywords: Asset Prices; Combination Forecasts; BVAR; FAVAR. JEL Codes: C11; C32; R53.

\section{Introduction}

The influence of financial markets on real economic activity and macroeconomic indicators has long been a subject of debate (see Stock \& Watson, 2003 for a review). The latest global economic downturn, sparked by the decline in house prices in the United States, has contributed to the recent revival of this discussion, particularly in the monetary policy domain. Espinoza et al. (2009) note that asset prices are determined in markets that are fundamentally forward looking, thus suggesting that these prices may act as leading indicators of macroeconomic variables and as such represent a group of possible predictors of future movements in the economy (Stock \& Watson, 2003). Financial markets are argued to influence economic activity in two important ways: financial and credit conditions influence firms' expansions and household expenditures; and asset prices contain expectations regarding firm profitability which is closely related to economic growth (Espinoza et al., 2009).

The relationship between asset prices and macroeconomic variables, specifically inflation and output growth, has been well documented in both economic theory and literature. The focus of these studies has, however, been placed on understanding this relationship with fewer studies assessing the usefulness of asset prices as potential predictors of inflation and output growth. Studies concentrating on the forecasting ability of asset prices have largely centralised on specific and individual asset prices that have been theoretically justified. These have included interest rates, exchange rates, house prices, term and default spreads. Stock and Watson (2003) provide a summary of this literature covering over

\footnotetext{
\# We would like to thank an anonymous referee for many helpful comments that improved the quality of the paper. However, any remaining errors are solely ours.

*Professor, University of Pretoria, Department of Economics, Pretoria, 0002, South Africa, Email: Rangan.Gupta@up.ac.za. Phone: +27 124203460.

** Graduate Student, Department of Economics, University of Pretoria. Contact details: Department of Economics, University of Pretoria, Pretoria, 0002, South Africa. Email: faaiqasalie@gmail.com.
} 
93 articles and working papers assessing the relationship between inflation or output growth and asset prices. Findings from the literature draw attention to the usefulness of asset prices in determining inflation and real output growth. These results, however, are plagued by problems of instability and in the case of output growth, low predictive ability. Stock and Watson (2003) conclude that these weak and unstable results may be due to the use of non-linear models or individual asset price variables. Furthermore, the review finds that often theoretically justified asset price predictors have limited predictive content empirically. Evidence from Rapach and Strauss (2005); and Stock and Watson (1999; 2003; 2004), among others, however suggests that assessing the predictive content of a group of asset prices may be more suitable as larger amount of information is captured. Thus, allowing for the explanation of a larger proportion of the variability in inflation and output growth. Much of the existing literature regarding the predictive ability of asset prices has been limited to developed countries such as the United States, European Union and the United Kingdom ${ }^{2}$ with little focus being placed on emerging and developing economies.

Stock and Watson (2003), which forms the foundation of this paper, assesses the short to medium term predictive content of asset prices in forecasting inflation and real output growth in the group of seven developed nations (G7) over the 1974 to 1983 and 1984 to 1999 periods. Findings from their study highlight that asset prices do aid in forecasting inflation and real output growth in some countries over some time periods, although the usefulness of asset prices are greater for forecasting inflation than real output growth. A second notable finding was that although forecasts using individual predictors were found to be unstable, combining these forecasts using simple techniques such as median and trimmed mean combining, circumvented many of the problems associated with individual predictor models and in most cases improved the accuracy of inflation and real output growth forecasts. Stock and Watson (2003) find simple forecast combining methods to be reliably superior to the benchmark AR model in addition to being low cost and relatively easy to perform.

As mentioned before, the literature focusing on developing economies is particularly thin and South Africa is no exception to this finding. ${ }^{3}$ The majority of studies for South Africa have focused on identifying the best models for forecasting inflation and real GDP growth without identifying the best predictors of these movements. The role of individual variables in forecasting inflation and output growth have furthermore concentrated on theoretically identified variables such as the interest rate and the term spread. Gupta and Kabundi (2010; forthcoming) study the usefulness of using large datasets to forecast macroeconomic variables, including inflation and real per capita growth (a measure of output), in South Africa. The results show that large scale models out perform or perform no worse than the small scale models considered (i.e. classical and Bayesian vector autoregressive models and the Small Open Economy New Keynesian Dynamic Stochastic General Equilibrium model). Although the datasets considered contained over 200 variables, including financial sector indicators, no differentiation was made between the forecasting ability of individual variables or variable classes.

Data from South Africa seems to suggest that asset prices may indeed be a valuable source of information regarding future movements in inflation and real GDP growth. This is more clearly illustrated in Figure 1 and 2 below. Inflation movements seem to be well correlated with real market rates, although the correlation between inflation and the real term-spread is found to be weak. ${ }^{4}$ Other variables, such as producer prices and money supply indicators also share close correlations with

\footnotetext{
2 See Espinoza et al., 2009; Forni et al., 2003; Rapach \& Weber, 2004; Stock \& Watson, 2003; and Zaher, 2007.

3 See Aron and Muellbauer (2000; 2001); Fedderke and Schaling (2005); Kaseeram et al. (2004); Moolman (2002); and Nel (1996).

${ }^{4}$ The term-spread is here defined the long-term government bond yields less the short-term treasury bill rate.
} 
inflation. Similar, although weaker, dependencies are also observed between real GDP growth and asset prices.

Figure 1: Inflation and Asset Prices
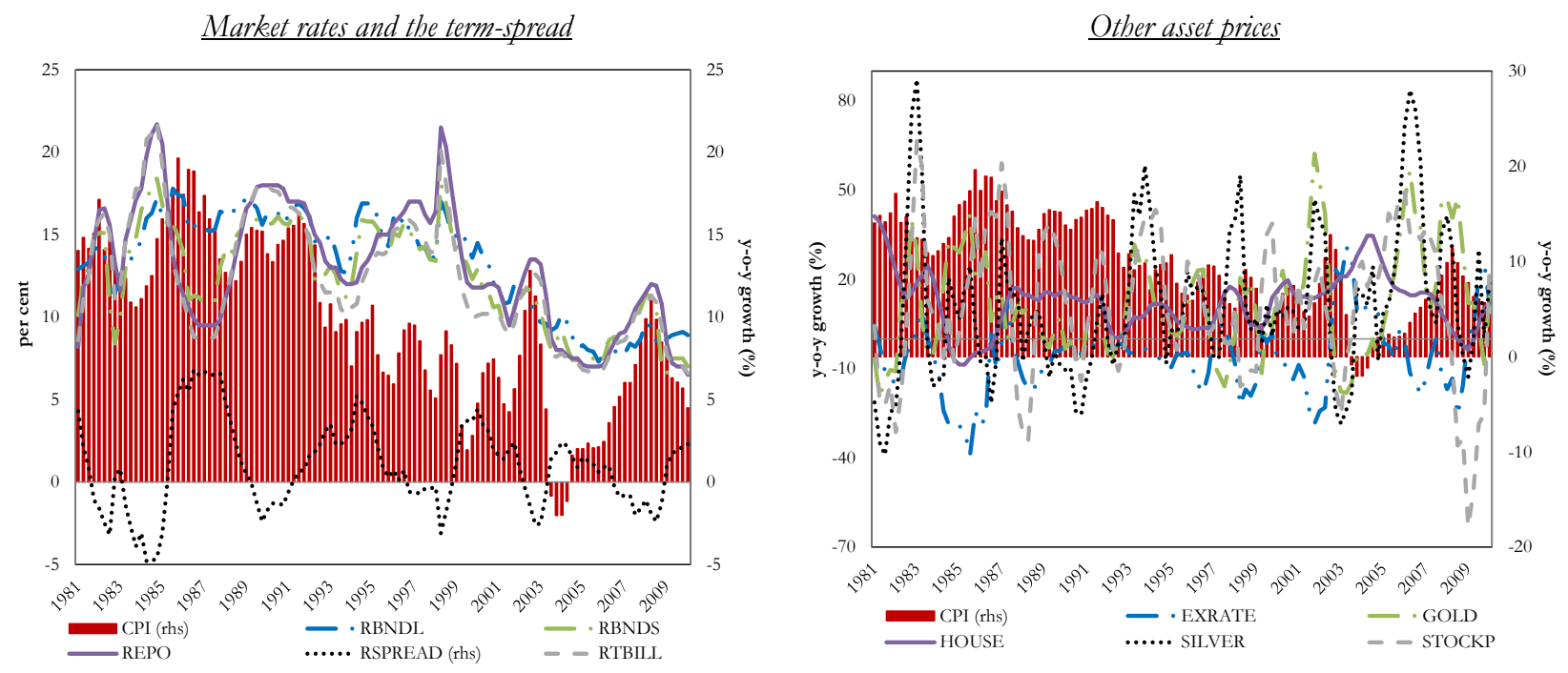

Figure 2: Real GDP growth and Asset Prices
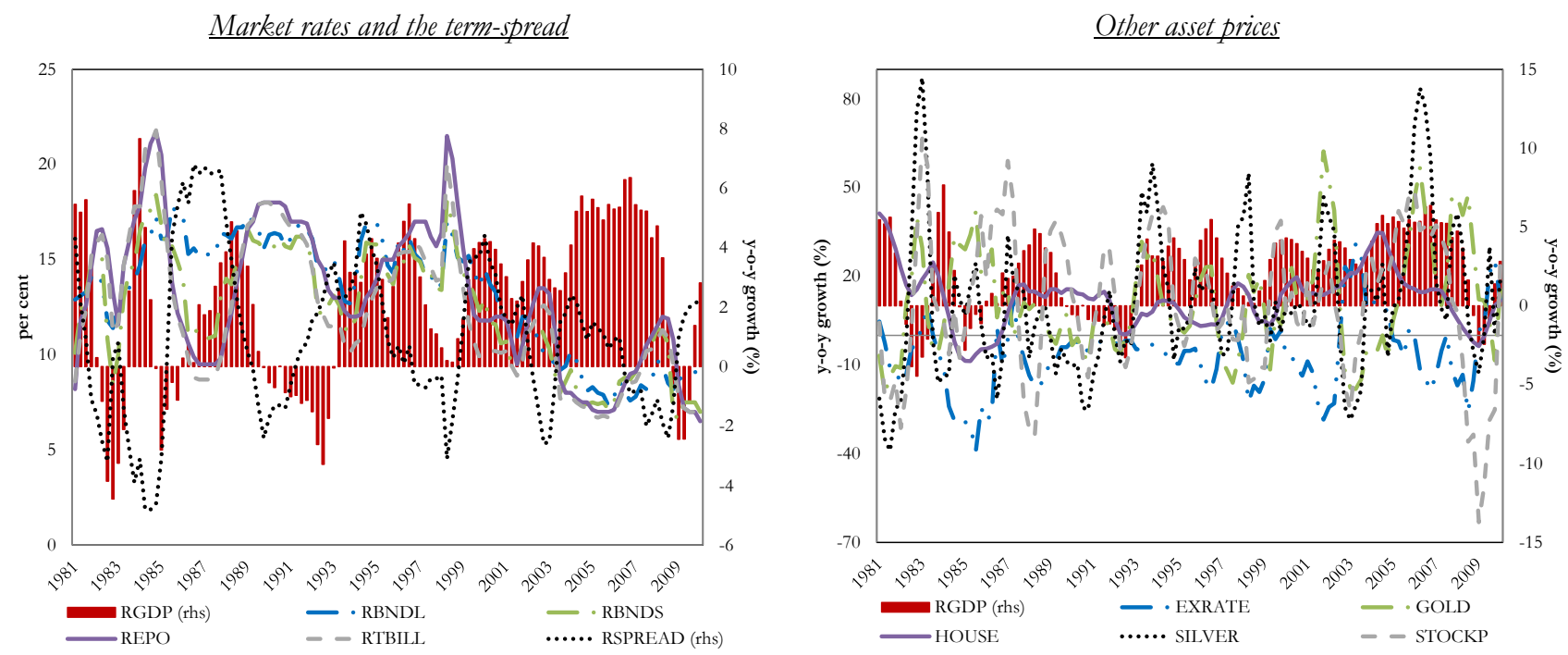

Accurate forecasting of inflation is particularly important in the case of an inflation targeting country such as South Africa. Improved inflation forecasts that take financial market influences into account will support monetary policy and improve the effectiveness of policy rates, which have a lagged impact, as a monetary policy tool. Forecasts of real GDP growth are important in any country and more so in South Africa where high levels of unemployment have been a persistent and prolonged problem. Over the past decade South Africa has experienced an unemployment rate of 26.6 per cent (World Bank), one of the highest in the world.

Following Stock and Watson (2003), this study aims to assess the forecasting potential of asset prices to predict inflation and real GDP growth for the case of South Africa. The individual and combined 
forecasting potential of 20 asset prices is assessed using pseudo out-of-sample forecasts for the period 2000:Q2 to 2010:Q2. The complete dataset includes 43 variables, 23 of which are non-asset price variables, spanning 1980:Q2-2010:Q2. The study uses both small and large data-set estimation methods. Specifically, autoregressive models, autoregressive distributive lagged models, forecast combinations, Bayesian vector autoregressive models as well as univariate and bivariate factor augmented vector autoregressive models with and without Bayesian restrictions are considered. In addition to contributing to the expansion of the literature on this topic and providing a South African specific case study, the following paper aims to aid in the task of forecasting inflation and real GDP growth by highlighting important leading indicators, providing an enriched understanding of the importance of the financial sector - which may lead to improvements in macroeconomic models; and contribute to the monetary policy debate regarding the importance of asset prices in designing monetary policies (Stock \& Watson, 2003). According to the knowledge of the authors, this is the first study to do so for the case of South Africa.

The remainder of the paper is organised as follows. Section 2 discusses the methodology and models considered. Section 3 briefly discusses the data employed and highlights the transformations undertaken. Section 4 looks at the measures used to compare model performances while Section 5 discusses the findings of the paper. Section 6 concludes with a summary of the main findings.

\section{Methodology}

\subsection{Assessing the Impact of Individual Variables}

In evaluating the potential forecasting ability of asset variables in estimating inflation and real GDP growth respectively, this study follows the methodology employed by Stock and Watson (2003). ${ }^{5}$ Individual Autoregressive Distributive Lagged (ARDL), or transfer function, models are used to estimate the relationship between the dependent, lagged dependent and a potential predictor variable (including its lags). These models take the form of equation (eq.) 1.

$$
y_{t+h}^{h}=\beta_{0}+\beta_{1}(L) y_{t}+\beta_{2}(L) X_{t}+u_{t+h}^{h}
$$

This model is used to estimate step ahead forecasts of inflation and real GDP growth. Thus, $y_{t+h}^{h}$ is equivalent to $\frac{1}{h} \sum_{j=1}^{h} \Delta y_{t-j}$ and represents the h-step ahead forecast of the dependent variable. $y_{t+h}^{h}$ is linear in both the potential predictor variables (i.e. $X_{t}$ ) as well as the lagged dependent variable. $\beta_{0}$ is a constant. $\beta_{1}$ denotes the impact of the lagged dependent variable, where $(L) y_{t}$ is a lagged polynomial of the dependent variable. $\beta_{2}$ captures the impact of the potential predictor variable and its lags illustrated as $(L) X_{t}$; where $(L) X_{t}$ is a lagged polynomial of the potential predictor variable. $u_{t+h}^{h}$ is an error term. For the estimation of inflation, 42 ARDL models are generated for the 42 explanatory variables. In the case of real GDP growth forecasts, 41 ARDL models are generated. The output gap is not included as a dependent variable in the latter ARDL models.

The models generated are used to conduct one to eight step-ahead out-of-sample forecasts of inflation and real GDP growth. These forecasts are then compared to the respective benchmark

\footnotetext{
${ }^{5}$ Inflation and real GDP growth are also referred to as the variables of interest or interested variables.
} 
models which take the form of a pure autoregressive (AR) model. AR models estimate the dependent variable, here inflation and real GDP growth, using lagged values of itself. The number of lags included is determined using the Schwarz Information Criteria (SIC). Specifically, lag lengths of two and one were used for the inflation and real GDP growth benchmark models. The form of the benchmark model is the same as in eq.1, where $\beta_{2}=0$.

Following Gupta and Kabundi (2010); Stock and Watson (2003; 2004); and Zaher (2007), among others, the paper further analyses whether the use of large scale models may out-perform these individual and benchmark forecasts. Large scale models tend to produce superior forecasts as they allow for the inclusion of more variables and hence more information. This also removes the need for subjective decision making regarding the inclusion of variables and decreases the problem of omitted variable bias. In this paper three primary methods of forecasting inflation and real GDP growth using large data sets are considered. These methods include Forecast Combinations, Bayesian Vector Autoregressions (BVAR) and Factor Augmented Vector Autoregressions (FAVAR). Specifically, four types of FAVARs are considered, namely Univariate and Multivariate FAVARs with and without Bayesian restrictions. These models are discussed in more detail below.

\subsection{Assessing the Impact of Variable Groups}

\subsubsection{Forecast Combinations}

Forecast combining is considered to be a useful technique for "....sharing strengths of different forecasting procedures..." (Yang, 2004:205) and is an alternative method of imposing "....structure on high-dimensional forecasting models" (Stock \& Watson, 2004:1). Empirical as well as theoretical evidence (see Clemen \& Winkler, 1986; Rapach \& Strauss, 2005; 2010; Stock \& Watson, 2003; 2004; and Yang, 2004) indicates that forecast combining generally improves the predictive ability of models as it includes more variables or potential predictors, thus increasing the amount of information used in generating forecasts and capturing demand, supply and policy dimensions. Four primary forecast combination methods are used in this study. These include Simple, Discount Mean Squared Forecast Errors (MSFE), Cluster and Principal Components combinations. According to Rapach and Strauss (2010), these combination methods can generally be described by eq.2:

$$
\hat{y}_{C B, t+h \mid t}^{h}=\sum_{i=1}^{n} w_{i, t} \hat{y}_{i, t+h \mid t}^{h}
$$

where $\hat{y}_{C B, t+h \mid t}^{h}$ is the combined forecast of the variable of interest and $w_{i, t}$ is the weight of the individual ARDL forecasts, $\hat{y}_{i, t+h \mid t}^{h}$, in the combined forecast.

\section{Simple Combinations}

Unlike the other methods of combining, simple combinations do not take into account the historical performance of the individual ARDL forecast models (Stock \& Watson, 2003; 2004) and as a result they do not require a "hold-out" period for calculating the weights (Rapach \& Strauss, 2010). Three simple combination methods are considered in this study, namely the mean, median and trimmed mean. Mean combinations are the weighted summation of the forecasts whereby all individual 
forecasts receive an equal weighting, i.e. $w_{i, t}=\frac{1}{n}$ (where $\mathrm{n}$ is the number of individual ARDL forecasts). The sample median of the individual ARDL models is used for the median combination. Trimmed mean combinations excludes the lowest and highest individual forecasts. The remaining forecasts are aggregated with a weight of $w_{i, t}=\frac{1}{(n-2)}$ assigned to each forecast (Rapach \& Strauss, 2005; 2010).

\section{Discount MSFE Combinations}

Discount MSFE combinations allows the modeller to place more value on the recent historical performance of the individual ARDL forecasts which is measured using the MSFEs (Rapach \& Strauss, 2010). The weights used in this combination process (see eq. 3) are dependent on the discount factor (i.e. $\delta$ ). As $\delta$ decreases, the weight attributed to the recent historical forecasts increases. A discount factor of one reduces the combination method to the Bates and Granger (1969) optimal combination forecast when the individual forecasts are uncorrelated (Stock \& Watson, 2004). Following Stock and Watson (2004) we use $\delta=1.0$ and $\delta=0.9$.

$$
w_{i, t}=\frac{m_{i, t}^{-1}}{\sum_{j=1}^{n} m_{j, t}^{-1}},
$$

where: $m_{i, t}=\sum_{s=T_{0}}^{t-h} \delta^{t-h-s}\left(Y_{s+h}^{h}-\hat{Y}_{i, s+h \mid s}^{h}\right)^{2} ; 0 \leq \delta \leq 1$.

\section{Cluster Combinations}

Cluster combinations or combining, as developed by Aiolfi and Timmermann (2006), is a conditional combining approach which incorporates information about the forecast persistence and historical performance of individual models (Rapach \& Strauss, 2010). In this study, we specifically employ the Previous Best conditional combination strategy. Clusters are formed per estimated period from the initial holdout out-of-sample period to the end of the out-of-sample period. Individual model forecasts are grouped into clusters ( $\mathrm{k}$ ) based on their MSFE values. The first cluster contains the forecasts with the lowest MSFEs. Each cluster of forecasts is then averaged and the clusters with the lowest MSFEs are included in the combination model. The optimal weights for the combination model are estimated using ordinary least squares (OLS) based on these clusters (Aiolfi \& Timmermann, 2006). Following Aiolfi and Timmermann (2006) we consider $\mathrm{k}=2$ and $\mathrm{k}=3$.

\section{Principal Components Combinations}

The principal component method of forecast combining comprises the extraction of the principal components $(p)$ from the individual ARDL forecasts. These components are then used to form a regression in which the weights are estimated using OLS. This method differs from dynamic factor and factor augmented vector autoregression models in that the factors are estimated from the panel of forecasts and not the actual variables.

$$
y_{s+h}^{h}=\phi_{1} \hat{p}_{1, s+h \mid s}^{h}+\ldots+\phi_{m} \hat{p}_{m, s+h \mid s}^{h}+v_{s+h}^{h}
$$


The combination forecast is then simply the aggregation of the principal components multiplied by their estimated weights. Principal components are linearly estimated from the individual ARDL forecasts such that each $p$ captures the variability and information contained in the individual forecasts. The first $p$ explains most of the variability followed by the second, third and so on (Chan et al., 1999; Rapach \& Strauss, 2010; Stock \& Watson, 2004). The number of components included in the study was based on the ICp3 criterion as suggested by Bai and Ng (2002).

\subsubsection{Vector Autoregressions (VAR)}

'Atheoretical' VAR models initially suggested by Sims (1980) is an “...n-equation, n-variable linear model in which each variable is in turn explained by its own lagged values, plus current and past values of the remaining $n-1$ variables" (Stock \& Watson, 2001:1). Formally, following Sims (1980), eq. 5 illustrates an unrestricted VAR model where $y$ is a $n \times 1$ vector of variables estimated. Unlike with the ARDL models where the dependent variable is forecast by h-step ahead projections, VAR models estimate multistep forecasts by iterating the VAR forward (Stock \& Watson, 2002). $y_{t}$ is therefore equivalent to $\Delta y_{t}+A(L) \Delta y_{t}$.

$$
y_{t}=A_{0}+A(L) y_{t}+\varepsilon_{t}
$$

$A_{0}$ is $n \times 1$ a vector of constant terms, $A(L)$ is a $n \times n$ polynomial matrix of lagged $y_{t}$ with lag length $p$. Using the SIC, a maximum lag length of 4 was identified for each variable. Thus, the initial four quarters of the sample period (i.e. 1980:Q2 to 1981:Q1) are used to feed the lags. $\varepsilon_{t}$ is a $n \times 1$ vector of error terms. Following Sims (1980) $\varepsilon_{t}$ is assumed to be normally distributed with a mean of zero and constant variance.

The main advantage of VAR models is that they allow one to simultaneously model and capture the dynamics of multiple data time series within a single system. VAR models, however, have been criticised for problems of low dimensionality and over-paramatisation, thus restricting the analyst or policy maker in terms of the number of variables that can be included in the model and possibly leading to omitted variable bias. Generally, VAR models consist of only six to eight variables. Bernanke et al. (2005) highlights that limitations to the number of variables included in the analyses leads to problems of contaminated results which causes price and other puzzles; subjective choices regarding variables to include and the representation of these variables; and restrictions in terms of the impacts that can be analysed as one can only assess the impacts of the variables included. For these reasons, BVAR models as suggested by Litterman (1981); Doan et al. (1984); Todd (1984); Litterman (1986); and Spencer (1993) as well as FAVAR models proposed by Bernanke et al. (2005); and Ahmadi and Ritschl, (2007) are considered in this paper. These methodologies resolve the lowdimensional problem associated with VAR models while enabling the continued use of the dynamic system framework (Gupta \& Kabundi, 2010).

\section{(i) Bayesian Vector Autoregressions (BVAR) ${ }^{6}$}

In addition to resolving the over-paramitisation problem associated with VAR models, BVAR estimation enables the use of prior knowledge of the distribution of variables. Ahmadi and Ritschl (2007) note this to be a logical step when the data used is historical and not estimated. These

${ }^{6}$ This paper follows the econometric framework of the BVAR model described in Gupta et al. (forthcoming). 
restrictions are imposed through hyper-parameters on the distribution of the variables coefficient, thus providing the relationship between the system parameters (Gupta et al., forthcoming).

This study follows the work of Gupta et al. (forthcoming) which is based on Litterman (1986) where the mean of the distribution of potential predictor variables, their lags and the lags of the interested variable are set to zero, suggesting that they are less important. The mean of the first lag of the interested variable is usually set to one. This restriction is based on the assumption that the lag of the interested variable impacts its future movements. The prior expectation is further imposed by specifying the standard deviation of the variable distribution. This indicates the certainty or uncertainty with which the prior mean is imposed. The larger the standard deviation, the greater the uncertainty regarding whether the prior mean is equal to the true mean. The standard deviation is assumed to decrease as the lag increases denoting greater confidence that the coefficient of the lagged variable will equal its prior mean value of zero. Specifying standard deviations eliminates the problem of deciding on the optimal number of lags to include. Following Litterman (1986), the standard distributions of lags of the dependent are larger than those of the other variables. A diffuse prior distribution is assumed for the constant term (Gupta \& Kabundi,2010; forthcoming; Gupta et al., 2011; forthcoming; and Litterman, 1986).

Formally, the variable distributions are generally $\beta_{i} \square N\left(1, \sigma_{\beta_{i}}^{2}\right)$ for the first lag of the variable of interest and $\beta_{j} \square N\left(0, \sigma_{\beta_{j}}^{2}\right)$ for potential predictor variables and their lags. These priors are also known as the 'Minnesota priors' and assume that the variables used contain a unit-root. Given that all our data is stationary, we assume the value of the first lag dependent to also be zero as done by Banbura et al. (2010) and Gupta et al. (forthcoming). Hence the variable distributions used in this paper are specifically $\beta_{i} \square N\left(0, \sigma_{\beta_{i}}^{2}\right)$ and $\beta_{j} \square N\left(0, \sigma_{\beta_{j}}^{2}\right)$.

The standard deviations employed are estimated using the formula proposed by Doan et al. (1984). The significance of this formula is that it allows for the estimation of a large number of coefficient variances using only a small number of hyper-parameters (Gupta \& Kabundi, forthcoming; and Gupta et al., forthcoming). Specifically these hyper-parameters are $w, d$ and the weighting matrix, $f(i, j)$. The formula for the standard deviation prior, $S(i, j, m)$, imposed on variable $j$ in equation $i$ at lag $m$ is specified in by. 6 .

$$
S(i, j, m)=\left[w^{*} g(m) * f(i, j)\right] \frac{\hat{\sigma}_{i}}{\hat{\sigma}_{j}}
$$

$f(i, j)=1$, if $i=j$ and $k_{i j}$ otherwise, with $\left(0 \leq k_{i j} \leq 1\right)$, and $g(m)=m^{-d}$, with $d>0 . \hat{\sigma}_{i}$ is the estimated standard error of the univariate autoregression for the $i^{\text {th }}$ variable and similarly $\hat{\sigma}_{j}$ is the estimated standard error of the univariate autoregression for the $j^{\text {th }}$ variable. According to Gupta et al. (forthcoming), the measurement units of the variables are not a problem when specifying the prior as the ratio $\frac{\hat{\sigma}_{i}}{\hat{\sigma}_{j}}$ accounts for any differences. The hyper-parameter, $w$, measures the overall tightness of the prior which increases as $\mathrm{w}$ declines. The $g(m)$ parameter quantifies the tightness on lag $m$ relative to lag one. This parameter has a decaying factor, $d$, which ensures that the prior tightens as $m$ increases. The parameter $f(i, j)$ captures the relationship between the tightness of the $j^{\text {th }}$ variable or potential predictor variables in equation $i$ relative to the $i^{\text {th }}$ variable (i.e. the lagged dependent 
variables). The prior becomes tighter as $k_{i j}$ decreases.

Five alternative sets of hyper-parameters are considered in this paper. These range from $w=0.3$ and $d=0.5$ to $w=0.1$ and $d=2$. The certainty of the coefficient means increases across the alternatives considered (i.e. the standard deviation of priors decrease). The model achieving the lowest relative MSFE is illustrated and reported on in Section 5. In the case of this study, these hyper-parameters were $w=0.3$ and $d=0.5$, in line with the standard Minnesota-type priors and those used by Gupta and Kabundi (2010; forthcoming) and Gupta et al. (2011). $k_{i j}$ is set to 0.5 .

\section{(ii) Factor Augmented Vector Autoregressions $(F A V A R)^{7}$}

FAVAR models are a combination of VAR and Dynamic Factor Models (DFM) that allow for the inclusion of many variables through the use of factors. Specifically, this technique minimises the size of the original dataset to a few factors while retaining the important information contained in the original dataset. Factors are useful in that they eliminate the problem of measurement bias often associated with macroeconomic data series. FAVAR models differ from PC combinations as the factors are extracted from the macroeconomic data series as opposed to the ARDL forecasts. Unlike the BVAR approach, FAVAR models do not require the implementation of restrictions although provision is made in the model specification for the inclusion of prior knowledge where variables are added directly in addition to the factors considered. This study estimates FAVAR models including and excluding Bayesian restrictions (Bernanke et al., 2005; and Ahmadi \& Ritschl, 2007). These restrictions follow those used in the BVAR estimation.

FAVAR models can be estimated using two alternative approaches, specifically the one-step and twostep approach (see Bernanke et al., 2005). This paper follows the two-step approach, which requires the extraction of the factors using principal component analyses and then using these factors, also known as the unobserved data, along with the observed data to estimate the FAVAR. In this paper the observed data refers to inflation and real GDP growth in the multivariate case (i.e. MFAVAR and MBFAVAR) and inflation or real GDP growth only in the univariate model (i.e. UFAVAR and UBFAVAR). The use of multivariate and univariate models in this context is to test the significance of including real GDP growth in the case of inflation, and vice-versa, as a specific and observable variable.

Since the FAVAR model is a mere combination of the DFM and VAR models, the first step is to estimate the DFM model. Individual time series are expressed in the DFM as the sum of the common unobserved component consisting of a small number of common factors and an idiosyncratic component for each variable. As suggested by Biovin and $\mathrm{Ng}$ (2005), the idiosyncratic component (i.e. error term) is estimated using an $\mathrm{AR}(\mathrm{p})$ process. Following Gupta et al. (forthcoming), this is illustrated in by. 7 .

$$
X_{t}=\lambda P_{t}+\xi_{t}
$$

$X_{t}$ equals the standardised version of $Y_{t}$, a $n \times 1$ covariance stationary process $Y_{t}=\left(y_{1 t}, \ldots, y_{n t}\right)^{\prime}$; $P_{t}$ represents a $r \times 1$ vector of static factors, $\lambda$ equals an $n \times r$ matrix of factor loadings, and $\xi_{t}$ equals a $n \times 1$ vector of idiosyncratic components. $P_{t}$ and $\xi_{t}$ are mutually orthogonal stationary

\footnotetext{
${ }^{7}$ This paper follows the econometric framework of the FAVAR model described in Bernanke et al. (2005).
} 
processes, while $X_{t}=\lambda P_{t}$ equals the common component. The representation of $X_{t}$ as a function of only $P_{t}$ does not limit the explanation of $X_{t}$ being related to past values of $P_{t}$ as $P_{t}$ in itself may be interpreted as including the lags of itself.

The common component of the DFM is an unobserved component and therefore requires estimation. Following Stock and Watson (2002), the static principal component (PC) approach is used on $X_{t}$ to extract the factors. The test proposed by Alessi et al., (2010), which is more suitable than the Bai and $\mathrm{Ng}$ (2002) test for a dataset with relatively small number of variables, suggested three factors for the whole data set, while, the same indicated that two and one factors should be extracted from the non-asset and the asset blocks respectively. Once the DFM is estimated, the extracted factors are combined with the VAR model to generate the FAVAR process. Following Bernanke et al. (2005), the FAVAR model takes the following form:

$$
\left[\begin{array}{l}
\hat{Y}_{t+h} \\
\hat{P}_{t+h}
\end{array}\right]=\Phi(L)\left[\begin{array}{l}
Y_{t} \\
P_{t}
\end{array}\right],
$$

where $\hat{Y}_{t+h}$ and $\hat{P}_{t+h}$ represent vectors including the variable of interest and the extracted factors respectively. $\Phi(L)$ is a lag polynomial of the variables of interest and factors.

\section{Data}

A group of 26 variables were collected for this study following Stock and Watson (2003). This group is comprised of eleven asset prices, four activity indicators, six money supply indicators and four variables capturing wages, goods and commodity price movements. As explained by Stock and Watson (2003:792-800), the variables included were done so for analytical completeness and due to their empirical validity in explaining inflation and real GDP growth both in developed and developing economies. These variables were primarily collected from the South African Reserve Bank, Statistics South Africa, the IMF International Financial Statistics database, the Global Financial Database and Bloomberg. The data was collected over the period 1980:Q2 to 2010:Q2. The period 1980:Q2 to 1999:Q4 was used as the in-sample estimation period, while the post-inflation targeting period, starting in 2000:Q1 to 2010:Q2 is used for out-of-sample forecasting. The end point of the sample is driven by the availability of data at the time the paper was being written. Note, the parameter estimates of the model was recursively updated over the out-of-sample period to yield, 42 one-stepahead forecasts, 41 two-step-ahead forecasts and so on until 35 eight-step-ahead forecasts. Additional data series were created using the above mentioned data. As a result a total number of 43 variables were utilised in the study. Output and inflation were used as the dependent variables. A list of the variables is displayed in Table 1.

A number of transformations were completed on the data in the following order. The first transformation involved the removal of seasonal or cyclical variations from the data. The U.S. Census Bureau's X12 seasonal adjustment program was used for this purpose. Twenty-eight of the 43 data variables were seasonally adjusted. The second transformation was to ensure that all data had the same frequency, in this case quarterly. Daily and monthly data were therefore linear averaged to obtain a quarterly figure. Following Stock and Watson (2003) both nominal and real variables were 
Table 1: Variable description

\begin{tabular}{|c|c|c|}
\hline $\begin{array}{r}\text { Variable } \\
\text { Asset Prices }\end{array}$ & Data frequency & Description \\
\hline EXRATE & Q & Nominal effective exchange rate \\
\hline DIVPR & M, Q & Johannesburg Stock Exchange Dividend Yield (\%) \\
\hline GOLD & $\mathrm{D}, \mathrm{Q}$ & Gold price (per ounce, $\mathrm{R}$ ) \\
\hline HOUSE & M, Q & ABSA House price index - All homes (smoothed, R) \\
\hline RBNDL & M, Q & Government bond yield, 10 years and over $(\%)$ \\
\hline RBNDS & M, Q & Government bond yield, $0-3$ years $(\%)$ \\
\hline REPO & Q & Policy rate $(\%)$ \\
\hline REXRATE & M, Q & Real effective exchange rate \\
\hline RGOLD & Q & Real gold price (per ounce, R): GOLD/CPI*100 \\
\hline RHOUSE & Q & Real ABSA House price index: HOUSE/CPI*100 \\
\hline RRBNDL & Q & Government bond yield, 10 years and over (\%): RBNDL-inflation \\
\hline RRBNDS & Q & Government bond yield, $0-3$ years (\%): RBNDS-inflation \\
\hline RREPO & Q & Real policy rate: REPO-inflation \\
\hline RRTBILL & Q & Real treasury bill rate (\%): RTBILL-inflation \\
\hline RSILVER & Q & Real silver price (per ounce, $\mathrm{R}):$ SILVER/CPI*100 \\
\hline RSPREAD & Q & Term spread: RBNDL-RTBILL \\
\hline RSTOCKP & Q & Real JSE stock price index: STOCKP/CPI*100 \\
\hline RTBILL & Q & Treasury Bill Rate $(\%)$ \\
\hline SILVER & $\mathrm{D}, \mathrm{Q}$ & Silver price (per ounce, $\mathrm{R}$ ) \\
\hline STOCKP & Q & JSE stock prices index \\
\hline \multicolumn{3}{|c|}{ Activity Indicators and Commodity Prices } \\
\hline CAPU & Q & Capacity utilization: RGDP/potential RGDP*100 \\
\hline COMMOD & Q & Economist Commodity Price Index \\
\hline CPI & $\mathrm{M}, \mathrm{Q}$ & Consumer Price Index \\
\hline EARN & Q & Real remuneration per worker in the non-agricultural sectors \\
\hline EMP & Q & Employment Index - non-agricultural sectors \\
\hline EMP_GAP & Q & Employment Index - non-agricultural sectors: EMP-potential EMP \\
\hline IP & M,Q & Industrial Production Index \\
\hline IP_GAP & Q & Industrial Production Index: IP - potential IP \\
\hline OIL & $\mathrm{D}, \mathrm{Q}$ & Crude oil price (per barrel, R) \\
\hline PGDP & Q & GDP deflator: nominal GDP/RGDP*100 \\
\hline PPI & M, Q & Producer Price Index \\
\hline RCOMMOD & Q & Real Economist Commodity Price Index: COMMOD/CPI*100 \\
\hline RGDP & $\mathrm{Q}$ & Real Gross Domestic Product $(\mathrm{R} \mathrm{m})$ \\
\hline RGDP_GAP & Q & Real Gross Domestic Product (R m): RGDP-potential RGDP \\
\hline ROIL & Q & Crude oil price (per barrel, R): OIL/CPI*100 \\
\hline \multicolumn{3}{|l|}{ Money supply } \\
\hline M0 & M,Q & Monetary aggregates - M0 $(\mathrm{R} \mathrm{m})$ \\
\hline M1 & $\mathrm{M}, \mathrm{Q}$ & Monetary aggregates - M1 $(\mathrm{R} \mathrm{m})$ \\
\hline M2 & M,Q & Monetary aggregates - M2 ( $\mathrm{R} \mathrm{m})$ \\
\hline M3 & M,Q & Monetary aggregates - M3 ( $\mathrm{R} \mathrm{m})$ \\
\hline RM0 & Q & Real monetary aggregates - M0 (R m): M0/CPI*100 \\
\hline RM1 & Q & Real monetary aggregates - M1 $(\mathrm{R} \mathrm{m}): \mathrm{M} 1 / \mathrm{CPI} * 100$ \\
\hline RM2 & Q & Real monetary aggregates - M2 $(\mathrm{R} \mathrm{m}): \mathrm{M} 2 / \mathrm{CPI} * 100$ \\
\hline RM3 & Q & Real monetary aggregates - M3 $(\mathrm{R} \mathrm{m}): \mathrm{M} 3 / \mathrm{CPI} * 100$ \\
\hline
\end{tabular}

used. The use of nominal and real variables ensures that all the explanatory power from these 
variables is included. Real variables were obtained by deflating the individual nominal series using the consumer price index. Some variables were also logarithmically transformed to ensure a more normal, smooth distribution and enable the modelling of variables in terms of growth rates. The JSE dividend price yield, real term spread, long and short term government bond yields, the repurchase rate, shortterm Treasury bill rates and real GDP gap variables were left in their level form. Lastly, variables were tested for stationarity. The main test employed was the Dickey-Fuller test with GLS de-trending (DFGLS). The DF-GLS test is used instead of the standard Dickey-Fuller or Augmented Dickey Fuller tests as it has been proven to be more robust when faced with variance breaks (Cook, 2004) and has stronger testing power (Elliot et al., 1996). ${ }^{8}$ The Kwiatkowski-Phillips-Schmidt and Shin (KPSS) and Phillips-Perron (PP) tests were also applied to support the results from the DF-GLS tests. ${ }^{9}$ Nonstationary variables were differenced and in some cases the difference from potential or natural estimates (i.e. gaps) were generated to ensure that all variables were stationary. Potential variables were estimated using the Hodrick-Prescott (HP) filter with a penalty of 1600 - the standard penalty for quarterly data (Hodrick \& Prescott, 1997). A detailed list of transformations as well as data sources is provided in Appendix A, while Appendix B provides the summary statistics of the variables used in this study.

Three data blocks are referred to in the forecast combining and large-scale models. These blocks are all variables, asset variables and non-asset variables. The purpose of this division is to specifically test the significance of asset price variables relative to the other data blocks in forecasting inflation and real GDP growth. The non-asset variable group comprises the activity indicators, money supply indicators as well as the variables capturing wages, goods and commodity price movements. A total of 12 high-dimensional models are estimated for each of the three data blocks, resulting in a total of 36 high dimensional models. In addition to the benchmark model, 42 and 41 ARDL models are estimated for inflation and real GDP growth respectively. Each model estimates eight-step-ahead forecasts of which the predictive power of the one, two, four, six and eight horizons are presented in Tables 2 to 5.

\section{Measuring model forecast performance}

In assessing the forecasting accuracy of the 36 forecasting models estimated this paper largely follows the methodology of Stock and Watson (2003), who uses the Mean Squared Forecasting Error (MSFE) as a measure of forecast performance. The MSFE of each model is calculated and the relative MSFE approach is used to compare competing models to the benchmark AR model as well as with each other. This approach was chosen as it is both familiar and easy to interpret. The relative MSFE ratio is represented by eq. 9 .

$$
\operatorname{MSFE}_{\text {ratio }}=\frac{\frac{1}{T_{2}-T_{1}-h+1} \sum_{t=T_{1}}^{T_{2}-h}\left(Y_{t+h}^{h}-\hat{Y}_{i, t+h \mid t}^{h}\right)^{2}}{\frac{1}{T_{2}-T_{1}-h+1} \sum_{t=T_{1}}^{T_{2}-h}\left(Y_{t+h}^{h}-\hat{Y}_{0, t+h \mid t}^{h}\right)^{2}}
$$

$Y_{t+h}^{h}$ is the actual data at $t+h, \hat{Y}_{0, t+h \mid t}^{h}$ indicates the benchmark model forecast of $Y$ h-steps ahead; and $\hat{Y}_{i, t+h \mid t}^{h}$ represents competing model forecasts of $Y$ h-steps ahead. A MSFE ratio of less than one

\footnotetext{
${ }^{8}$ Testing power refers to the power to reject the null hypothesis when it is false.

9 The unit root test results are available on request.
} 
indicates that the competing model produces a superior forecast of inflation or real GDP growth, in terms of accuracy, relative to the benchmark model. A MSFE ratio of larger than one, however, suggests that the benchmark model produces a more accurate forecast with a lower forecasting error.

In addition to the relative MSFE, an alternative test of model forecasting ability is also considered. Specifically, the paper uses the $\mathrm{HLN}_{\mathrm{h}}$ forecast encompassing test to evaluate the forecasting ability of asset price models, and hence asset prices, relative to competing data block models. The forecast encompassing approach is chosen as it allows one to assess whether a specific data block contains information useful for forecasting, beyond that contained in the competing model (Rapach \& Strauss, 2010). Forecast encompassing techniques are argued to be a more powerful measure of forecasting ability than relative MSFE (Rapach \& Weber, 2004). Given the extensive number of models considered, this study only analyses the best asset based models (identified in Section 5) relative to the best non-asset and all variable data block models.

The test for encompassed forecasts considers the construction of an optimal forecast using a convex combination of two rival forecasts as illustrated by eq. $10 . \hat{y}$ is the respective model forecast of inflation or real GDP growth, $0 \leq \lambda \leq 1$ and $\mathrm{cm}$ represents each of the competing models (i.e. benchmark, non-asset and all variable models).

$$
\hat{y}_{\text {opt }, t+h \mid t}^{h}=\lambda_{\text {asset }} \hat{y}_{\text {asset }, t+h \mid t}^{h}+\lambda_{c m} \hat{y}_{c m, t+h \mid t}^{h},
$$

The forecast encompassing measure tests whether $\lambda_{\text {asset }}=0$ or $\lambda_{\text {asset }}>0$. In the event that $\lambda_{\text {asset }}=0$, information contained in the asset based models are considered to be encompassed by the $\mathrm{cm}$, indicating that asset price models do not provide any additional information to that contained in the competing model. If $\lambda_{\text {asset }}>0$, however, asset prices provide additional useful information for forecasting inflation and real GDP growth. Harvey et al. (1998) developed the $\mathrm{HLN}_{\mathrm{h}}$ test statistic which tests the null hypothesis of $\lambda_{\text {asset }}=0$ against the one-sided upper-tail alternative, $\lambda_{\text {asset }}>0$. The Harvey et al. (1998) test statistic is illustrated by eq.11.

$$
H L N_{h}=\left[\hat{V}\left(\bar{d}^{h}\right)\right]^{-1 / 2} \bar{d}^{h},
$$

where: $\quad \bar{d}^{h}=P_{h}^{-1} \sum_{t=R+P_{0}}^{T-h} \hat{d}_{t+h \mid t}^{h}$

$$
\begin{aligned}
& \hat{d}_{t+h \mid t}^{h}=\left(\hat{u}_{a s s e t, t+h \mid t}^{h}-\hat{u}_{j, t+h \mid t}^{h}\right) \hat{u}_{a s s e t, t+h \mid h^{h}}^{h}\left(\hat{u}_{k, t+h \mid t}^{h} \text { is the forecasting error of model k=asset, cm }\right) \\
& \hat{V}\left(\bar{d}^{h}\right)=P_{h}^{-1}\left(\hat{\omega}_{o}+2 \sum_{j=1}^{h-1} \hat{\omega}_{j}\right) \\
& \hat{\omega}_{j}=P_{h}^{-1} \sum_{t=R+P_{0}+j}^{T-h}\left(\hat{d}_{t+h \mid t}^{h}-\bar{d}^{h}\right)\left(\hat{d}_{(t-j)+h \mid(t-j)}^{h}-\bar{d}^{h}\right)
\end{aligned}
$$

Clark and McCracken (2001) argue that when forecasting models are nested, many of the standard test statistics (including the $\mathrm{HLN}_{\mathrm{h}}$ test) are no longer asymptotically Gaussian, as the forecast errors are asymptotically the same and thus perfectly correlated. They provide an alternative test for nested models, called the ENC-T test. This test is employed to evaluate the forecasting accuracy of the best and second best asset block models relative to the benchmark AR model. The test statistic is defined as follows: 


$$
E N C-T=(P-1)^{1 / 2} \frac{\bar{C}}{\left(P^{-1} \sum_{t=R}^{T-1}\left(c_{t+h}-\bar{C}\right)\right)^{1 / 2}}
$$

where, $c_{t+h}=\hat{v}_{0, t+h}\left(\hat{v}_{0, t+h}-\hat{v}_{1, t+h}\right)$ and $\bar{c}=\sum_{t=R}^{T-1} c_{t+1}, R$ denotes the estimation period, $P$ is the prediction period, $f$ is some generic loss function $\left(f\left(v_{0, t+h}\right)=v_{0, t+h}^{2}\right.$, in our case), $h \geq 1$ is the forecast horizon, $\hat{v}_{0, t+h}$ and $\hat{v}_{1, t+h}$ are $h$-step ahead prediction errors for models 0 and 1 (where model 0 is the best model on average within the asset block), constructed using Newey and West (1987) type consistent estimators.

The hypotheses of interest are:

$$
\begin{gathered}
H_{0}: E\left(f\left(v_{0, t+h}\right)-f\left(v_{1, t+h}\right)\right)=0, \text { and } \\
H_{A}: E\left(f\left(v_{0, t+h}\right)-f\left(v_{1, t+h}\right)\right)>0 .
\end{gathered}
$$

The limiting distribution is $N(0,1)$ for $h=1$. The limiting distribution for $h>1$ is non-standard, as discussed in Clark and McCracken (2001). As long as a Newey and West (1987) type estimator is used when $h>1$, however, then the tabulated critical values closely approximate the $N(0,1)$ values (Bhardwaj and Swanson, 2006).

\section{Results}

This section is divided into three parts. Section 5.1 and 5.2 provides a detailed analysis of the estimation results for inflation and real GDP growth respectively. Tables 2 and 4 summarises the relative MSFE results of the individual ARDL models for the forecast horizons considered, while Tables 3 and 5 indicate the relative MSFE results for the forecast combinations and large scale data models. From these sections, the best and second best models for forecasting inflation and real GDP growth are identified using the MSFE criteria. The forecasting ability of the best and second best asset price models are then further tested using the forecast encompassing tests discuss in Section 4. The results from these tests are discussed in Section 5.3 and summarised in Tables 6 and 7.

\subsection{Inflation}

Results from the combined individual ARDL forecasts indicate that asset prices are useful for forecasting inflation, particularly over the short-term (i.e. over one or two horizons). Table 3 shows that at almost every horizon regardless of the forecast combining method used, combined asset price forecasts are superior to the benchmark model, obtaining a maximum reduction of 27.0 per cent in the forecasting error. This lead, however, decreases as the time horizon extends further into the future - in line with the findings of Stock and Watson (2003). On average across combining methods, combined asset price forecasts are 10.9 per cent superior to the benchmark model in the short term, 6.5 per cent in the medium term and 3.5 per cent in the long term. This decline in reduction in the forecasting error suggests that asset prices contain more information about short to medium term movements relative to long-term or structural changes. This is in line with expectations as these variables capture market conditions and sentiment, which are generally short to medium term 
Table 2: Out-of-Sample Forecasting Performance of ARDL Models for Inflation

\begin{tabular}{|c|c|c|c|c|c|}
\hline \multicolumn{6}{|c|}{$\underline{\text { Ratios }}$} \\
\hline Asset Variables & $h=1$ & $h=2$ & $h=4$ & $h=6$ & $h=8$ \\
\hline Benchmark model* & 0.007 & 0.009 & 0.012 & 0.010 & 0.009 \\
\hline DIVPR & 1.030 & 1.053 & 1.115 & 1.093 & 1.126 \\
\hline DLEXRATE & 0.781 & 0.802 & 0.801 & 0.872 & 0.908 \\
\hline DLGOLD & 0.882 & 0.820 & 0.876 & 0.921 & 0.973 \\
\hline DLHOUSE & 1.000 & 1.018 & 1.012 & 1.001 & 1.037 \\
\hline DRBNDL & 1.082 & 1.005 & 0.980 & 0.968 & 1.022 \\
\hline DRBNDS & 1.050 & 0.995 & 0.975 & 0.980 & 1.053 \\
\hline DREPO & 1.038 & 1.009 & 1.039 & 1.014 & 1.038 \\
\hline DLREXRATE & $0.770^{* *}$ & $0.795^{* *}$ & $0.800 * *$ & $0.862 * *$ & $0.906^{* *}$ \\
\hline DLRGOLD & 0.882 & 0.821 & 0.876 & 0.922 & 0.973 \\
\hline DLRHOUSE & 1.000 & 1.017 & 1.010 & 1.001 & 1.036 \\
\hline DRRBNDL & 1.072 & 1.019 & 0.904 & 0.943 & 1.035 \\
\hline DRRBNDS & 1.079 & 0.987 & 0.930 & 0.945 & 0.979 \\
\hline DRREPO & 1.052 & 0.997 & 0.969 & 0.951 & 0.985 \\
\hline DRRTBILL & 1.034 & 0.992 & 0.965 & 0.936 & 0.985 \\
\hline DLRSILVER & 0.898 & 0.938 & 0.966 & 1.015 & 1.000 \\
\hline RSPREAD & 0.997 & 1.012 & 1.085 & 1.100 & 1.092 \\
\hline DLRSTOCKP & 1.020 & 0.959 & 0.915 & 0.939 & 0.964 \\
\hline DLSILVER & 0.897 & 0.937 & 0.966 & 1.014 & 1.000 \\
\hline DRTBILL & 1.039 & 1.010 & 1.034 & 1.009 & 1.068 \\
\hline DLSTOCKP & 1.020 & 0.959 & 0.915 & 0.938 & 0.964 \\
\hline \multicolumn{6}{|c|}{ Non-Asset Variables } \\
\hline CAPU & 1.023 & 0.998 & 0.992 & 0.980 & 0.952 \\
\hline DLCOMMOD & 1.010 & 1.011 & 1.022 & 1.026 & 1.025 \\
\hline DLEARN & 1.004 & 1.003 & 0.991 & 1.003 & 1.007 \\
\hline DLEMP & 3.428 & 7.291 & 2.682 & 3.905 & 5.383 \\
\hline EMP_GAP & 1.090 & 1.116 & 1.041 & 1.004 & 1.341 \\
\hline DLIP & 0.948 & 0.969 & 0.941 & 0.937 & 1.034 \\
\hline IP_GAP & 1.070 & 1.021 & 1.019 & 1.040 & 1.045 \\
\hline DLM0 & 0.903 & 0.914 & 0.923 & 0.947 & 0.967 \\
\hline DLM1 & 0.893 & 0.866 & 0.887 & 0.955 & 0.920 \\
\hline DLM2 & 1.009 & 0.956 & 0.939 & 0.976 & 1.010 \\
\hline DLM3 & 1.029 & 0.954 & 0.959 & 0.998 & 0.978 \\
\hline DLOIL & 0.912 & 0.943 & 0.949 & 0.966 & 1.048 \\
\hline DLPGDP & 0.974 & 1.011 & 0.971 & 0.970 & 0.952 \\
\hline DLPPI & 1.104 & 0.960 & 0.966 & 0.876 & 1.059 \\
\hline DLRCOMMOD & 1.010 & 1.011 & 1.022 & 1.026 & 1.025 \\
\hline DLRGDP & 1.059 & 1.004 & 0.986 & 0.996 & 1.099 \\
\hline RGDP_GAP & 1.115 & 1.047 & 1.020 & 0.986 & 0.956 \\
\hline DLRM0 & 0.907 & 0.919 & 0.928 & 0.951 & 0.970 \\
\hline DLRM1 & 0.894 & 0.867 & 0.889 & 0.955 & 0.921 \\
\hline DLRM2 & 1.018 & 0.956 & 0.936 & 0.980 & 1.006 \\
\hline DLRM3 & 1.026 & 0.955 & 0.960 & 1.000 & 1.031 \\
\hline DLROIL & 0.911 & 0.943 & 0.949 & 0.966 & 1.048 \\
\hline $\begin{array}{l}\text { Results in bold } \\
\text { * The result for } \\
\text { ** Indicates the }\end{array}$ & $\begin{array}{l}\text { ate } A R D \\
\text { given } b \\
g A R D L\end{array}$ & $\begin{array}{l}\text { s that out } \\
F E(\%)\end{array}$ & he bench & del & \\
\hline
\end{tabular}


Table 3: Out-of-Sample Forecasting Performance of Large-Scale Models and Forecast Combination Methods for Inflation

\begin{tabular}{|c|c|c|c|c|c|}
\hline Forecast Combination: Mean & $h=1$ & $\begin{array}{l}\text { Ratio } \\
\quad h=2\end{array}$ & $h=4$ & $h=6$ & $h=8$ \\
\hline - All Variables & 0.900 & 0.954 & 0.917 & 0.962 & 1.011 \\
\hline - Asset Variables & 0.894 & 0.904 & 0.904 & 0.938 & 0.978 \\
\hline - Non-Asset Variables & 0.921 & 1.005 & 0.934 & 0.989 & 1.047 \\
\hline \multicolumn{6}{|l|}{ Forecast Combination: Median } \\
\hline - All Variables & 0.957 & 0.956 & 0.950 & 0.962 & 0.999 \\
\hline - Asset Variables & 0.953 & 0.956 & 0.949 & 0.962 & 0.999 \\
\hline - Non-Asset Variables & 0.952 & 0.947 & 0.939 & 0.961 & 0.994 \\
\hline \multicolumn{6}{|c|}{ Forecast Combination: Trimmed Mean } \\
\hline - All Variables & 0.932 & 0.935 & 0.927 & 0.972 & 1.017 \\
\hline - Asset Variables & 0.926 & 0.932 & 0.927 & 0.968 & 1.022 \\
\hline - Non-Asset Variables & 0.957 & 0.950 & 0.934 & 0.981 & 1.020 \\
\hline \multicolumn{6}{|c|}{ Forecast Combination: Discounted MSFE $(\delta=1.0)$} \\
\hline - All Variables & 0.891 & 0.927 & 0.917 & 0.965 & 1.013 \\
\hline - Asset Variables & 0.885 & 0.900 & 0.902 & 0.939 & 0.977 \\
\hline - Non-Asset Variables & 0.917 & 0.960 & 0.937 & 0.995 & 1.050 \\
\hline \multicolumn{6}{|c|}{ Forecast Combination: Discounted MSFE $(\delta=0.9)$} \\
\hline - All Variables & 0.888 & 0.919 & 0.914 & 0.964 & 1.014 \\
\hline - Asset Variables & 0.881 & 0.900 & $0.898 * *$ & 0.938 & 0.978 \\
\hline - Non-Asset Variables & 0.913 & 0.943 & 0.936 & 0.992 & 1.052 \\
\hline \multicolumn{6}{|c|}{ Forecast Combination: Cluster $(\mathrm{k}=2)$} \\
\hline - All Variables & 0.890 & 0.896 & 0.914 & 1.002 & 1.055 \\
\hline - Asset Variables & 0.863 & 0.879 & 0.921 & 0.958 & 1.010 \\
\hline - Non-Asset Variables & 0.912 & 0.920 & 0.915 & 1.065 & 1.143 \\
\hline \multicolumn{6}{|c|}{ Forecast Combination: Cluster $(\mathrm{k}=3)$} \\
\hline - All Variables & 0.846 & 0.899 & 0.908 & 1.034 & 1.106 \\
\hline - Asset Variables & 0.849 & 0.895 & 0.929 & 0.969 & 1.012 \\
\hline - Non-Asset Variables & 0.911 & 0.909 & 0.913 & 0.975 & 1.241 \\
\hline \multicolumn{6}{|c|}{ Forecast Combination: Principle Components } \\
\hline - All Variables & $0.730^{* *}$ & $0.815^{* *}$ & 1.036 & 0.944 & 0.918 \\
\hline - Asset Variables & 0.798 & 0.838 & 1.046 & 0.942 & $0.846^{* *}$ \\
\hline - Non-Asset Variables & 0.904 & 1.046 & 1.148 & 1.016 & 1.029 \\
\hline \multicolumn{6}{|l|}{ Bayesian Vector Autoregression } \\
\hline - All Variables & 1.113 & 1.242 & 1.491 & 1.544 & 1.658 \\
\hline - Asset Variables & 1.015 & 0.905 & 0.930 & 0.965 & 0.997 \\
\hline - Non-Asset Variables & 1.126 & 1.278 & 1.400 & 1.472 & 1.532 \\
\hline \multicolumn{6}{|c|}{ Univariate Factor Augmented Vector Autoregression } \\
\hline - All Variables & 1.105 & 0.895 & 0.941 & 1.006 & 0.996 \\
\hline - Asset Variables & 0.947 & 0.908 & 0.929 & $0.918^{* *}$ & 0.933 \\
\hline - Non-Asset Variables & 0.827 & 0.833 & 0.966 & 0.996 & 0.989 \\
\hline \multicolumn{6}{|c|}{ Univariate Bayesian Factor Augmented Vector Autoregression } \\
\hline - All Variables & 0.872 & 0.846 & 0.918 & 1.005 & 1.021 \\
\hline - Asset Variables & 0.895 & 0.952 & 0.970 & 0.987 & 1.001 \\
\hline - Non-Asset Variables & 0.841 & 0.879 & 0.966 & 1.022 & 1.037 \\
\hline \multicolumn{6}{|c|}{ Multivariate Factor Augmented Vector Autoregression } \\
\hline - All Variables & 1.127 & 0.891 & 0.951 & 1.009 & 0.998 \\
\hline - Asset Variables & 0.959 & 0.937 & 0.944 & 0.924 & 0.938 \\
\hline - Non-Asset Variables & 0.830 & 0.831 & 0.974 & 1.001 & 0.995 \\
\hline \multicolumn{6}{|c|}{ Multivariate Bayesian Factor Augmented Vector Autoregression } \\
\hline - All Variables & 0.867 & 0.845 & 0.918 & 1.005 & 1.021 \\
\hline - Asset Variables & 0.891 & 0.952 & 0.969 & 0.984 & 0.998 \\
\hline - Non-Asset Variables & 0.837 & 0.877 & 0.967 & 1.025 & 1.037 \\
\hline
\end{tabular}

concepts. Relative to other data blocks, however, asset prices provide the most accurate forecasts of inflation over the longer term. 
Overall, the results highlight that principal component (PC) combining produces the most accurate forecasts of inflation over the out-of-sample forecast horizon. In the overall best and second best models, the forecasting error is reduced by 11.2 (all variable block) and 10.6 per cent (asset price block) relative to the benchmark model. As mentioned in Section 2.2.1 the PC model is comprised of three, two and one principal component(s) in the case of the all variable, non-asset price and asset price models. The identification of PC as the best and second best forecast combination method suggests that the use of actual variables results in the inclusion of redundant information thereby weakening the forecasting ability of the respective models. Forecast combining, as with FAVAR analysis, removes this complication by extracting only the valid information. Inflation forecasts using PC combining are up to 27.0 per cent superior relative to those produced by the benchmark model.

With regards to the non-asset data block, simple combination methods are found to produce the most accurate forecasts of inflation. Specifically, median forecast combinations yield the largest reductions in forecasting errors. These reductions are however small, averaging 3.1 per cent over the estimation period. Forecast combinations based on non-asset price variables only are generally found to achieve the smallest gains in forecasting accuracy. On average across horizons and combination methods, MSFE reductions of only 1.8 per cent are achieved when using the non-asset price block, relative to 7.1 and 5.6 per cent when using asset price and all variable blocks respectively.

Discounted MSFE forecast combinations, using a $\delta=0.9$, produce the second most accurate inflation forecasts across all data blocks considered, improving forecasting accuracy by $6.0,8.1$ and 3.3 per cent (relative to the benchmark model) for the all variable, asset price and non-asset price data blocks respectively. When using only asset price variables, discounted MSFE forecast combinations produce their most optimal and consistent forecasts, achieving gains of just over 10.0 per cent in the short to medium term and 4.1 per cent in the longer run. Given that inflation is generally hard to predict in the long run, these improvements in the forecasting error are noteworthy.

As with Rapach and Strauss (2005), and Stock and Watson (2003, 2004) simple combination forecasts were observed to be relatively stable, with median combinations consistently producing superior inflation forecasts across the forecast horizons. Simple combination methods therefore provide a lowcost methodology of achieving consistently superior forecasts of inflation relative to the benchmark model (Rapach \& Strauss, 2005; 2010; and Stock \& Watson, 2003; 2004). The magnitudes of the reductions in MSFE achieved are however in most cases the smallest (less than 5.0 per cent) amongst the competing combination models. Furthermore, combination forecasts using asset price variables only were found to yield the most stable forecasts of inflation, producing superior forecasts to the benchmark model at every horizon in four of the eight combining methods considered.

Setting the results from forecast combining aside, other large scale data set models considered, specifically the BVAR, UFAVAR, MFAVAR and their Bayesian counterparts, also show that asset price based estimations produce inflation forecasts superior to that of the benchmark model. On average, asset price based models reduce the forecasting error by between 3.8 and 7.3 per cent across the forecast horizon. Asset price based inflation forecasts are also found to be generally more consistent than those using other data blocks. Non-asset price based models are however observed to produce superior inflation forecasts, particularly in the short term. This is dissimilar to the results obtained from forecast combinations. BVAR models are the only exclusion to this finding. The inferior performance of other data blocks when using BVAR models, relative to FAVAR models, suggests that the use of factors rather that actual macroeconomic series may enhance the forecasting performance of models as they purge the model of any redundant or irrelevant information. This finding is in line with the prior identification of PC forecast combinations as the optimal combination 
method. Asset based FAVAR models generally also produce superior forecasts relative to the BVAR model, particularly over the short term. Despite being inferior, BVAR model forecasts highlight the significance of asset prices as predictors of future inflation. In fact, BVAR forecasts produced using asset prices only, are the only models to achieve superior forecasts to the benchmark model.

From the results, UFAVAR models are identified as producing the most accurate forecasts when only asset and non-asset prices are used. Respectively, the accuracy of inflation forecasts improves by 7.3 and 7.1 per cent over the out-of-sample forecast horizon. In the case of asset prices, this improvement in accuracy is consistent over the short, medium and long term with MSFE reductions of about 7.0 per cent being obtained. The superior accuracy of UFAVAR models relative to MFAVAR models suggests that the impact of real output growth may already be captured by the factors. This is confirmed by the results from the forecasts of output, discussed in Section 5.2, which identify nominal interest rates and real house prices as leading indicators of output growth. Relative to their classical equivalents, Bayesian restrictions only improve the short term forecasting ability of these models. This may indicate that over the medium to longer term, the unrestricted inclusion of the history of inflation becomes more important given the significant degree of persistence in inflation movements (Kaseeram et al., 2004). In the case of the all variable data block, however, restricted models produce the most accurate predictions of inflation. Bayesian restrictions in this case improves the forecasting accuracy of the model as the larger set of information included reduces the relative importance of past inflation rates. MBFAVAR and UBFAVAR models achieve similar levels of reductions in the forecasting error -7.1 and 7.0 per cent respectively.

Overall the findings suggest that across models, forecast combinations produce the most accurate forecasts of inflation. PC combination forecasts based on the all variable data block yields the most accurate forecasts of inflation over the forecast horizons. The 8.7 per cent improvement in forecasting accuracy achieved could be said to be largely driven by asset prices as independent asset price PC combinations reduces the relative MSFE by 8.1 per cent, whilst the non-asset model performs worse than the benchmark model. Inflation forecasts from the all variable PC model are unfortunately not consistently superior to the benchmark model. Specifically PC combined forecasts are out performed by the benchmark model at $\mathrm{h}=4$. The discounted MSFE $(\delta=0.9)$ approach where only asset prices are considered is identified as the second best inflation forecasting model. Unlike with the previous approach, this model produces a consistently superior forecast to the benchmark model, while achieving an 8.5 per cent improvement in forecasting accuracy. Results for best models per data block are provided in Table 6 .

With regards to specific asset prices, results from the individual ARDL models (see Table 3), indicate that most of the identified asset price variables provide superior forecasts of inflation relative to the benchmark model, although not consistently across all horizons. This puzzling result has also been highlighted by Stock and Watson (2003) and Zaher (2007). Forecast combining is used in an attempt to resolve this puzzle, which it does to a partial extent. On average, the improvements in forecasting accuracy range between 0.5 and 17.3 per cent. Real and nominal exchange rates and gold prices are the only two asset price variables that provide consistently superior forecasts of inflation relative to the benchmark model, with ARDL forecasts including exchange rates also producing superior forecasts relative to all large scale competing models over the medium to long term. The real exchange rate, which produces the most accurate forecast amongst all variables, reduces the MSFE by up to 21.8 per cent in the short run, 20.0 per cent in the medium term and 11.6 per cent over the long run. The substantial significance of the exchange rate and gold price in forecasting inflation in South Africa may be the result of the structure of the economy. South Africa is a mining and manufacturing exporting economy with a fairly large share of imported intermediary inputs. This makes the economy 
and hence inflation particularly vulnerable to changes in the exchange rate and mining commodity prices such as gold. Other asset price variables also highlighted as predictors of inflation include silver prices (as a short-term indicator) and the JSE Stock Price Index (over the medium to longer term). Real long and short-term government bond and short-term Treasury bill rates, as well as the repo rate were also found to be useful indicators although gains in forecasting accuracy are smaller. Little evidence is, however, found in support of the predictive content of the term spread for inflation. The term spread is found to produce superior forecasts in only the first horizon and even this improvement in forecasting accuracy is small at only 0.3 per cent (relative to the benchmark model). This is aligned with the empirical evidence of Stock and Watson (2003) as well as the literature review provided (Stock \& Watson, 2003).

With regards to non-asset price variables, M0 and M1 money supply indicators are identified as useful predictors of SA inflation, improving benchmark forecasts by 8.7 and 11.8 per cent over the short to medium term; and 4.3 and 6.2 per cent over the longer run. The output gap, capacity utilisation rate and oil prices are also particularly useful. Average earnings, surprisingly provide no additional information relative to benchmark forecasts despite rising concerns that excessive public sector wage increases may result in escalating price increases. The lack of evidence in support of commodity prices as an indicator of inflation may be the result of the indicator considered. The Economist Commodity Price Index was considered in this paper, which may not necessarily be a useful indicator of inflation for SA given the subcategories included.

\subsection{Real GDP Growth}

The relative MSFE results for real GDP growth forecasts, illustrated in Table 5, indicate that unlike the inflation results, asset price variables are less important for forecasting real GDP growth. Although forecasts based on asset prices are superior to those produced by the benchmark model, non-asset price variables predict more of the variability in real GDP growth. Results from the forecast combining exercise reveals that, on average, asset price forecasts are 7.8 per cent superior to the benchmark model while non-asset price variables improve forecasting accuracy by about 24.0 per cent. Forecast combination estimates of real GDP growth were generally observed to be consistently superior to those of the benchmark model.

Within the forecast combination models, PC combination forecasts using all variables are the most accurate, reducing the forecasting error by 40.5 per cent over the forecast horizon. The identification of PC combining (all variables) as the best combining method reinforces our findings from Section 5.1 that the use of components instead of actual variables removes any redundancies or irrelevant information from the model thereby allowing for more accurate forecasts. Over the short, medium and long term the forecasting error associated with the real GDP growth projections decline by 30.7, 33.9 and 53.7 per cent respectively. The rising trend in forecasting accuracy as the horizon extends into the future indicates not only the usefulness of large dataset forecasts but also highlights the weakness of the benchmark model in forecasting real GDP growth over the medium to longer term. The forecasting error of the benchmark model increases as the forecasting horizon extends into the future. Furthermore, the significance of the all variable PC combination as the best forecasting model indicates that although not when considered alone, asset prices are important in predicting real GDP growth when combined with non-asset prices. Independently asset and non-asset based PC forecast combination models reduce the forecasting error by 7.4 and 30.8 per cent respectively. However, when combined the reduction in the forecasting error improves to $40.5 \%$. 
Among combination forecast models using the asset price data block, cluster combination models $(\mathrm{K}=3)$ are identified as the most accurate forecasting model, improving estimates of real GDP growth by 10.8 per cent relative to the benchmark model. In the case of the non-asset data block, the optimal forecasting combination model identified is the discounted MSFE $(\delta=0.9)$, achieving a 35.3 per cent improvement in forecasting accuracy. This model is also the second most accurate forecast combination model. Similarly to the inflation result, simple combination methods although consistent and inexpensive produce relatively small gains in accuracy. On average, simple combination models reduce the forecasting error by between 6.8 and 14.3 per cent. The average reduction in the forecasting error across the forecast combinations considered is between 7.8 and 24.3 per cent.

In terms of specific data blocks in other large scale models, the MBFAVAR model is identified as the optimal forecasting model when using all variables or only non-asset variables. On average, these models reduce the forecasting error by 13.2 and 16.1 per cent, respectively. In the case of asset price variables, however, the optimal real GDP growth forecasting model is the MFAVAR, achieving a 12.2 per cent increase in forecasting accuracy relative to the benchmark model. The identification of multivariate models as superior to univariate models indicates that the direct inclusion of inflation in the FAVAR model improves the forecasts of real GDP growth. In the case of inflation, univariate models were generally found to outperform multivariate models. This is an interesting result and seems to suggest that inflation is a better predictor of real GDP growth, than real GDP growth is of inflation. Results from the individual ARDL models confirm this observation. On average, inflation improves real GDP growth forecasts by 43.4 per cent relative to the benchmark model. Real GDP growth, however, does not improve on benchmark forecasts of inflation. Neither do other indicators such as the real GDP gap, industrial production and capacity utilisation, which are considered to be measures of economic activity. The superior performance of the unrestricted asset price based FAVAR model relative to the restricted counterpart may be the result of asset prices being relatively weak indictors of real GDP growth. As a result the presence of past real GDP growth values improves the forecasts of models using only asset prices. The large amount of information in nonasset and all variable models, however, finds the presence of lagged real GDP growth as redundant, thus weakening their forecasts. The inclusion of restrictions in this case improves the forecasting accuracy of the MFAVAR models.

Results from FAVAR models indicate that non-asset price variables produce the most accurate forecasts of real GDP growth, with the inclusion of asset variables (i.e. the all variable case) reducing the forecasting ability of the model. BVAR models are the only exception to this finding. As with forecasts of inflation, asset based BVAR models are the only models to improve on benchmark forecasts. Overall, forecast combination models are once again identified as the most accurate predictors. Specifically, the best and second best real GDP growth forecasting models are identified as the PC combination model using all variables and the discounted MSFE combination $(\delta=0.9)$ of non-asset prices.

On average across data blocks, PC combination models are generally found to produce the most accurate and stable forecasts of inflation and real GDP growth over the short term. In the longer run, the evidence for the "best model methodology" is less clear. In the case of inflation, the UFAVAR produces the most accurate and stable forecasts on average, while in the case of real GDP growth, this is achieved by the Discounted MSFE $(\delta=0.9)$ combination model.

Individual ARDL results indicate that although most asset prices forecast real GDP growth more accurately than the benchmark model these gains are generally small and inconsistent over the forecast horizons considered (see Table 4). Relative to inflation, there are fewer asset prices that 
Table 4: Out-of-Sample Forecasting Performance of ARDL Models for Real GDP Growth

\begin{tabular}{|c|c|c|c|c|c|}
\hline \multicolumn{6}{|c|}{ Ratios } \\
\hline Asset Variables & $h=1$ & $h=2$ & $h=4$ & $h=6$ & $h=8$ \\
\hline Benchmark model* & 0.003 & 0.003 & 0.004 & 0.004 & 0.004 \\
\hline DIVPR & 0.990 & 1.031 & 1.199 & 1.006 & 1.048 \\
\hline DLEXRATE & 1.070 & 0.991 & 0.900 & 0.931 & 0.978 \\
\hline DLGOLD & 1.131 & 1.092 & 1.209 & 1.185 & 1.050 \\
\hline DLHOUSE & 0.819 & 1.009 & 1.105 & 1.214 & 1.314 \\
\hline DRBNDL & 1.006 & 0.810 & 0.622 & 0.751 & 0.916 \\
\hline DRBNDS & 0.941 & 0.742 & 0.573 & 0.557 & 0.737 \\
\hline DREPO & 0.886 & 0.784 & 0.741 & 0.782 & 0.829 \\
\hline DLREXRATE & 1.089 & 1.025 & 0.953 & 0.975 & 1.013 \\
\hline DLRGOLD & 1.115 & 1.377 & 1.220 & 1.198 & 1.033 \\
\hline DLRHOUSE & 0.741 & 0.824 & 0.999 & 0.766 & 0.951 \\
\hline DRRBNDL & 1.019 & 1.041 & 1.121 & 1.030 & 1.009 \\
\hline DRRBNDS & 1.020 & 1.034 & 1.099 & 1.014 & 1.019 \\
\hline DRREPO & 1.011 & 1.007 & 1.018 & 0.998 & 1.008 \\
\hline DRRTBILL & 1.034 & 1.029 & 1.025 & 1.002 & 1.016 \\
\hline DLRSILVER & 1.224 & 1.210 & 1.087 & 0.941 & 0.922 \\
\hline RSPREAD & 1.005 & 1.022 & 1.189 & 0.986 & 0.994 \\
\hline DLRSTOCKP & 0.974 & 0.996 & 0.927 & 0.871 & 0.927 \\
\hline DLSILVER & 1.274 & 1.323 & 1.242 & 1.048 & 1.083 \\
\hline DRTBILL & 1.012 & 0.828 & 0.778 & 0.745 & 0.870 \\
\hline DLSTOCKP & 1.001 & 0.931 & 0.979 & 0.939 & 0.981 \\
\hline \multicolumn{6}{|c|}{ Non-Asset Variables } \\
\hline CAPU & 0.842 & 0.844 & $0.364^{* *}$ & $0.133^{* *}$ & $0.055^{* *}$ \\
\hline DLCOMMOD & 0.553 & 0.712 & 1.107 & 1.013 & 0.887 \\
\hline DLCPI & 0.849 & 0.657 & 0.466 & 0.393 & 0.468 \\
\hline DLEARN & 1.001 & 1.022 & 1.011 & 0.972 & 1.011 \\
\hline DLEMP & 1.606 & 1.468 & 7.164 & 5.417 & 5.867 \\
\hline EMP_GAP & 1.087 & 1.469 & 1.825 & 1.966 & 2.008 \\
\hline DLIP & 0.988 & 1.009 & 1.134 & 0.980 & 0.984 \\
\hline IP_GAP & 0.893 & 0.755 & 0.645 & 0.573 & 0.670 \\
\hline DLM0 & 1.009 & 1.030 & 1.046 & 1.018 & 1.018 \\
\hline DLM1 & 1.025 & 1.015 & 0.954 & 0.944 & 0.995 \\
\hline DLM2 & 1.025 & 1.010 & 0.875 & 0.744 & 0.798 \\
\hline DLM3 & 1.012 & 1.015 & 0.966 & 0.888 & 1.027 \\
\hline DLOIL & 1.060 & 1.072 & 1.001 & 0.998 & 1.030 \\
\hline DLPGDP & 0.985 & 0.965 & 0.983 & 0.851 & 0.829 \\
\hline DLPPI & 1.054 & 0.934 & 0.801 & 0.749 & 0.747 \\
\hline DLRCOMMOD & $0.472^{* *}$ & $0.592^{* *}$ & 1.009 & 0.841 & 0.743 \\
\hline DLRM0 & 0.955 & 0.887 & 0.929 & 0.812 & 0.868 \\
\hline DLRM1 & 0.933 & 0.903 & 0.935 & 0.962 & 0.985 \\
\hline DLRM2 & 0.985 & 0.967 & 1.021 & 1.083 & 1.278 \\
\hline DLRM3 & 0.939 & 0.890 & 0.741 & 0.760 & 1.005 \\
\hline DLROIL & 1.046 & 1.064 & 1.013 & 1.006 & 1.029 \\
\hline
\end{tabular}

provide more accurate forecasts of real GDP growth (relative to the benchmark model). A small number of key predictors of economic activity have, however, been identified. These variables include real house prices (14.4 per cent more accurate than the benchmark model); nominal short and long term bond yields (29.0 and 17.9 per cent), short term nominal Treasury bill rates (15.3 per cent) and 
the nominal repurchase rate (19.5 per cent). Real house prices are a particularly useful short-term indicator, improving forecast accuracy by 21.7 per cent. This result is aligned with studies highlighting

Table 5: Out-of-Sample Forecasting Performance of Large-Scale Models and Forecast Combination Methods for Real GDP growth

\begin{tabular}{|c|c|c|c|c|c|}
\hline Forecast Combination: Mean & $h=1$ & $\begin{array}{l}\text { Ratio } \\
h=2\end{array}$ & $h=4$ & $h=6$ & $h=8$ \\
\hline - All Variables & 0.898 & 0.848 & 0.814 & 0.814 & 0.892 \\
\hline - Asset Variables & 0.963 & 0.905 & 0.876 & 0.881 & 0.949 \\
\hline - Non-Asset Variables & 0.845 & 0.805 & 0.775 & 0.765 & 0.846 \\
\hline \multicolumn{6}{|l|}{ Forecast Combination: Median } \\
\hline - All Variables & 0.982 & 0.937 & 0.892 & 0.912 & 0.955 \\
\hline - Asset Variables & 0.981 & 0.923 & 0.909 & 0.928 & 0.973 \\
\hline - Non-Asset Variables & 0.948 & 0.941 & 0.878 & 0.886 & 0.949 \\
\hline \multicolumn{6}{|c|}{ Forecast Combination: Trimmed Mean } \\
\hline - All Variables & 0.954 & 0.924 & 0.840 & 0.819 & 0.870 \\
\hline - Asset Variables & 1.012 & 0.970 & 0.897 & 0.890 & 0.930 \\
\hline - Non-Asset Variables & 0.915 & 0.903 & 0.809 & 0.770 & 0.822 \\
\hline \multicolumn{6}{|c|}{ Forecast Combination: Discounted MSFE $(\delta=1.0)$} \\
\hline - All Variables & 0.895 & 0.851 & 0.786 & 0.760 & 0.821 \\
\hline - Asset Variables & 0.963 & 0.921 & 0.888 & 0.887 & 0.950 \\
\hline - Non-Asset Variables & 0.846 & 0.802 & 0.719 & 0.675 & 0.727 \\
\hline \multicolumn{6}{|c|}{ Forecast Combination: Discounted MSFE $(\delta=0.9)$} \\
\hline - All Variables & 0.866 & 0.830 & 0.754 & 0.642 & 0.606 \\
\hline - Asset Variables & 0.954 & 0.915 & 0.884 & 0.880 & 0.946 \\
\hline - Non-Asset Variables & 0.804 & 0.773 & 0.675 & 0.518 & $0.466 * *$ \\
\hline \multicolumn{6}{|c|}{ Forecast Combination: Cluster $(\mathrm{k}=2)$} \\
\hline - All Variables & 0.835 & 0.789 & 0.797 & 0.741 & 0.846 \\
\hline - Asset Variables & 0.961 & 0.914 & 0.931 & 0.867 & 0.941 \\
\hline - Non-Asset Variables & 0.766 & 0.728 & 0.748 & 0.674 & 0.783 \\
\hline \multicolumn{6}{|c|}{ Forecast Combination: Cluster $(\mathrm{k}=3)$} \\
\hline - All Variables & 0.780 & 0.740 & 0.751 & 0.682 & 0.792 \\
\hline - Asset Variables & 0.912 & 0.876 & 0.898 & 0.839 & 0.934 \\
\hline - Non-Asset Variables & 0.715 & $0.651^{* *}$ & 0.710 & 0.593 & 0.583 \\
\hline \multicolumn{6}{|c|}{ Forecast Combination: Principle Components } \\
\hline - All Variables & $0.695 * *$ & 0.692 & 0.661 & 0.456 & 0.469 \\
\hline - Asset Variables & 0.825 & 0.809 & 0.968 & 1.173 & 0.855 \\
\hline - Non-Asset Variables & 0.793 & 0.825 & $0.530 * *$ & $0.391 * *$ & 0.924 \\
\hline \multicolumn{6}{|l|}{ Bayesian Vector Autoregression } \\
\hline - All Variables & 1.151 & 0.880 & 0.956 & 1.160 & 1.014 \\
\hline - Asset Variables & 0.928 & 0.806 & 0.867 & 0.971 & 1.033 \\
\hline - Non-Asset Variables & 1.345 & 0.882 & 0.941 & 1.123 & 1.011 \\
\hline \multicolumn{6}{|c|}{ Univariate Factor Augmented Vector Autoregression } \\
\hline - All Variables & 0.908 & 0.842 & 0.883 & 0.977 & 1.030 \\
\hline - Asset Variables & 0.936 & 0.846 & 0.891 & 0.979 & 1.021 \\
\hline - Non-Asset Variables & 0.741 & 0.766 & 0.847 & 0.960 & 0.986 \\
\hline \multicolumn{6}{|c|}{ Univariate Bayesian Factor Augmented Vector Autoregression } \\
\hline - All Variables & 0.849 & 0.787 & 0.867 & 0.960 & 0.993 \\
\hline - Asset Variables & 0.951 & 0.905 & 0.949 & 0.993 & 1.008 \\
\hline - Non-Asset Variables & 0.734 & 0.737 & 0.836 & 0.936 & 0.966 \\
\hline \multicolumn{6}{|c|}{ Multivariate Factor Augmented Vector Autoregression } \\
\hline - All Variables & 0.906 & 0.790 & 0.860 & 0.966 & 1.036 \\
\hline - Asset Variables & 0.929 & 0.772 & 0.787 & 0.911 & 0.992 \\
\hline - Non-Asset Variables & 0.775 & 0.782 & 0.843 & 0.955 & 0.983 \\
\hline \multicolumn{6}{|c|}{ Multivariate Bayesian Factor Augmented Vector Autoregression } \\
\hline - All Variables & 0.828 & 0.743 & 0.836 & 0.946 & 0.989 \\
\hline - Asset Variables & 0.901 & 0.816 & 0.870 & 0.957 & 0.997 \\
\hline - Non-Asset Variables & 0.737 & 0.734 & 0.824 & 0.932 & 0.967 \\
\hline
\end{tabular}

the use of house prices as a leading indicator of demand within the economy (Girouard \& Blöndal, 
2001). Bond yields, the short-term Treasury bill rate and the repurchase rate were found to be better indicators of medium to long term activity. Short and long term bond yields improve forecasts of real GDP growth by up to 42.7 and 37.8 per cent respectively whilst the short term Treasury bill and repurchase rate improved forecasts by up to 25.5 and 25.9 per cent. McCallum (1983) and Bernanke and Blinder (1992) highlight that interest rates are indicators of market expectations regarding future monetary policy and in turn, economic activity. Little evidence is, however, found for the term spread as a predictor of economic activity. ARDL models including the term spread improve real GDP forecasts on average by only 1.0 per cent over the long term with short to medium term forecasts being inferior to the benchmark model. Nel (1996) found the term structure of interest rates to be a significant indicator of SA economic activity over the 1974 to 1983 period, but over the 1984-1993 period the value of the term spread as a predictor of real GDP growth had decreased significantly. This is similar to Stock and Watson (2003) findings that of the seven countries considered only Canada and Japan show evidence of the term spread as an indicator of economic activity post-1984. Neither of these studies however consider the post-2000 period.

Individual non-asset price variables were also found to be useful predictors of real GDP growth. As with asset prices, however, many non-asset price variables did not provide consistent forecasts superior to the benchmark model. Four variables outperforming even the best large scale model were identified. These variables were real and nominal commodity prices, capacity utilisation and inflation. In the short run ARDL models, including either real or nominal commodity prices, achieves a 46.8 and 36.8 per cent decline in relative MSFE. This result is converse to the findings of Stock and Watson (2003) who found that commodity prices generally produce poorer forecasts than the benchmark model. The importance of the commodity price index in forecasting short term growth is indicative of the South African economy - a natural resource dependent country. Over the medium to longer term, capacity utilisation and inflation improve forecasting accuracy by up to 90.6 and 57.0 per cent, respectively (relative to the benchmark model). The industrial production gap, M3 money supply and producer price index were also found to be significant indicators, improving forecasting accuracy by more than 20.0 per cent in the medium to long term. An important observation from the individual ARDL model results is that the forecasting ability of the non-asset price variables identified improves as the forecast horizon increases. Employment and employment gap ARDL models perform the poorest followed by M2 money supply and oil prices.

\subsection{Forecast comparison tests}

In addition to evaluating the predictive ability of asset prices using the loss in relative MSFE, we also consider the $\mathrm{HLN}_{\mathrm{h}}$ and ENC-T tests. Results from these tests, discussed below, generally confirm the findings from the inflation and real GDP growth discussions. The results indicate that at certain horizons asset prices contain useful information, above that of the benchmark or competing model, for forecasting both inflation and real GDP growth. Table 6 and 7 summarises the HLN $\mathrm{H}_{\mathrm{h}}$ and ENC-T results for inflation and real GDP growth, respectively.

For inflation, the results from the forecast comparison tests are generally in line with the relative MSFE results, except where the asset model forecasts are compared to those of the benchmark model. Results from the ENC-T tests shows that at certain horizons the benchmark model encompasses the asset price based models. Looking at the results comparing the best asset price model with the benchmark model, it is observed that at $\mathrm{h}=4$ and $\mathrm{h}=6$, asset prices do not provide any additional information for forecasting inflation - contrary to the relative MSFE results which suggests that asset price models produce superior forecasts. Over the short and longer term, however, asset price models are demonstrated to contain statistically significant additional information for 
forecasting inflation. The results are less compelling when considering the second best asset model (i.e. MBFAVAR). The $\mathrm{HLN}_{\mathrm{h}}$ test also confirms the rankings of our asset price models. The results illustrate that in general the second best model is encompassed within the best model, while the best model is not encompassed in the second best model.

Table 6: ENC-T and forecast encompassing tests for inflation forecasting models

\begin{tabular}{|c|c|c|c|c|c|}
\hline \multicolumn{6}{|c|}{ Best and Second Best Asset Block Model relative to AR model } \\
\hline \multirow[t]{2}{*}{ Forecast Combinations: Principle Components } & $0.798 * *$ & $0.838^{* *}$ & 1.046 & 0.942 & $0.846^{* *}$ \\
\hline & $(0.23)$ & $(0.23)$ & $(0.01)$ & $(0.00)$ & $(0.24)$ \\
\hline \multirow[t]{2}{*}{ MBFAVAR } & $0.891^{*}$ & 0.952 & 0.969 & 0.984 & 0.998 \\
\hline & $(0.01)$ & $(0.01)$ & $(0.10)$ & $(0.01)$ & $(0.00)$ \\
\hline \multicolumn{6}{|c|}{ Best Asset Block Model relative to Best Non-Asset Block Model } \\
\hline \multirow[t]{3}{*}{ UFAVAR } & 0.965 & 1.006 & 1.082 & 0.946 & 0.856 \\
\hline & $(0.04)$ & $(0.00)$ & $(0.00)$ & $(0.05)$ & $(0.23)$ \\
\hline & {$[0.01]$} & {$[0.00]$} & {$[0.10]$} & {$[0.01]$} & {$[0.00]$} \\
\hline \multicolumn{6}{|c|}{ Best Asset Block Model relative to Best All Block Model } \\
\hline \multirow[t]{3}{*}{ Forecast Combinations: Principle Components } & 1.092 & 1.029 & 1.010 & 0.998 & 0.922 \\
\hline & $(0.01)$ & $(0.00)$ & $(0.00)$ & $(0.00)$ & $(0.10)$ \\
\hline & {$[0.08]$} & {$[0.03]$} & {$[0.00]$} & {$[0.00]$} & {$[0.00]$} \\
\hline \multicolumn{6}{|c|}{$\begin{array}{l}\text { Note: } * * *, * * * \text { indicate significance at } 10,5 \text { and } 1 \text { per cent based on the ENC-t test between the asset price models relative to the AR } \\
\text { models. } \\
\text { Values within } 0 \text { indicate the p-value for the forecast encompassing tests. That is we test whether in the case of the asset block, the best } \\
\text { (second best) model encompasses the second best (best) model against the one-sided, upper tail alternative hypothesis the best (second } \\
\text { best) model does not encompass the second best (best) model. For the other data blocks, the p-values in } 0 \text { test whether the best models } \\
\text { from the respective blocks are encompassed within the best model from the asset block. } \\
\text { The p-values in [] test whether the best model from the asset block is encompassed in the best models from the competing data blocks. }\end{array}$} \\
\hline
\end{tabular}

Table 7: ENC-T and forecast encompassing tests for real GDP growth forecasting models

\begin{tabular}{|c|c|c|c|c|c|}
\hline Ratio & $h=1$ & $h=2$ & $h=4$ & $h=6$ & $h=8$ \\
\hline \multicolumn{6}{|c|}{ Best and Second Best Asset Block Model relative to AR model } \\
\hline \multirow[t]{2}{*}{ MFAVAR } & 0.929 & $0.772^{* *}$ & $0.787^{* *}$ & 0.911 & 0.992 \\
\hline & $(0.00)$ & $(0.10)$ & $(0.10)$ & $(0.01)$ & $(0.03)$ \\
\hline \multirow[t]{2}{*}{ Cluster $\mathrm{k}=3$} & 0.912 & $0.876^{*}$ & $0.898^{*}$ & $0.839 * *$ & 0.934 \\
\hline & $(0.00)$ & $(0.03)$ & $(0.02)$ & $(0.10)$ & $(0.05)$ \\
\hline \multicolumn{6}{|c|}{ Best and Second Best Asset Block Model relative to Best and Second Best Non-Asset Block Model } \\
\hline \multirow[t]{3}{*}{ Discounted MSFE $(\delta=0.9)$} & 1.155 & 0.998 & 1.165 & 1.760 & 2.130 \\
\hline & $(0.01)$ & $(0.00)$ & $(0.01)$ & $(0.00)$ & $(0.00)$ \\
\hline & {$[0.11]$} & {$[0.00]$} & [0.12] & [0.70] & {$[0.75]$} \\
\hline \multicolumn{6}{|c|}{ Best and Second Best Asset Block Model relative to Best and Second Best All Block Model } \\
\hline \multirow[t]{3}{*}{ Discounted MSFE $(\delta=0.9)$} & 1.072 & 0.929 & 1.044 & 1.419 & 1.637 \\
\hline & $(0.00)$ & $(0.10)$ & $(0.00)$ & $(0.00)$ & $(0.00)$ \\
\hline & {$[0.08]$} & {$[0.00]$} & {$[0.02]$} & {$[0.55]$} & {$[0.65]$} \\
\hline \multicolumn{6}{|c|}{$\begin{array}{l}\text { Note: } *, * *, * * \text { indicate significance at } 10,5 \text { and } 1 \text { per cent based on the ENC-t test between the asset price models relative to the AR } \\
\text { models. } \\
\text { Values within } 0 \text { indicate the p-value for the forecast encompassing tests. That is we test whether in the case of the asset block, the best } \\
\text { (second best) model encompasses the second best (best) model against the one-sided, upper tail alternative hypothesis the best (second } \\
\text { best) model does not encompass the second best (best) model. For the other data blocks, the p-values in } 0 \text { test whether the best models } \\
\text { from the respective blocks are encompassed within the best model from the asset block. } \\
\text { The p-values in [ test whether the best model from the asset block is encompassed in the best models from the competing data blocks. }\end{array}$} \\
\hline
\end{tabular}

Relative to other data blocks the results show that asset prices do contain useful additional information for forecasting inflation. At the 10.0 per cent significance level the PC asset model is not encompassed by the competing data block models. Over the short to medium forecast horizon, the 
competing data block models are also not encompassed in the PC asset model suggesting that each model contains information that may enhance the forecasts of inflation. In the longer run, at $h=8$, the ENC-T and $\mathrm{HLN}_{\mathrm{h}}$ test results indicate that the PC asset model contains significant information for forecasting inflation, more so than the benchmark or competing models. This confirms the findings from the relative MSFE results which show that asset price based models produce the most accurate forecasts of inflation in the longer run (when compared to competing models).

The forecast comparison results for models predicting real GDP growth also indicate that at certain horizons asset prices contain useful information beyond that supplied by competing models. This is the case when compared to both the benchmark and competing data block models. Relative to the results for inflation, however, asset based forecasting models are not as useful in forecasting real GDP growth. The $\mathrm{HLN}_{\mathrm{h}}$ results indicate that across most horizons, forecasts produced by the nonasset Discounted MSFE $(\delta=0.9)$ model includes information provided by the MFAVAR asset price model. At $\mathrm{h}=2$, however, the MFAVAR asset model does provide additional information that improves the forecast of real GDP growth. Relative to the all variable Discounted MSFE $(\delta=0.9)$ model, however, the PC asset price model in the short to medium term is not encompassed by the all variable model (10.0 per cent significance level). This is an important observation as it confirms that despite all variable data block forecasts achieving a larger reduction in MSFE over the short to medium term, asset price models still contain useful information for forecasting real GDP growth.

The overall results from the forecast comparison tests highlight a critical observation regarding the importance of asset prices in forecasting both inflation and real GDP growth. This observation is that although forecasting models based on asset prices (only) do not always provide the most accurate forecasts, they are useful as they introduce information that may not have been otherwise included. This information may not consistently be important to the forecast, but does enable forecasting models to better predict future movement as a larger and more varied set of indicators are included.

\section{Conclusion}

This study aimed to assess the forecasting ability of asset prices in predicting inflation and real GDP growth for South Africa. The results indicate that although asset prices are more useful for forecasting inflation, they still play an important role in predicting real GDP growth at certain periods. The finding that asset prices occasionally provide additional information highlights the limitations of standard variables generally considered. Asset prices allow for the inclusion of additional financial market information which has become increasingly important to economies today. Furthermore asset price data are readily and more importantly timely available, making it a valuable source of information. Forecasters are thus able to include the most recent data in their predictions, allowing for more accurate forecasts.

Our findings are largely aligned with the empirical literature. In addition to proving the importance of asset prices, this study also highlights the convenience of combination forecasts. Similarly to Rapach and Strauss $(2005,2008)$; and Stock and Watson (2003; 2004), we find that forecast combinations produce more accurate forecasts of inflation and real GDP growth than the benchmark AR model, individual ARDL models and other more sophisticated competing models. Specifically, PC combination forecasts including all variables are observed to produce the most accurate forecasts of both inflation and real GDP growth. This is closely followed by the Discounted MSFE $(\delta=0.9)$ approach. Classic and Bayesian FAVAR models are also found to yield superior forecasts to BVAR models. Das et al. (2010), Gupta et al. (forthcoming), Leamer (2007); and Zaher (2007) find similar 
results. The identification of PC combinations and FAVAR models suggests that the use of factors improves forecasting ability as they circumvent problems of redundant or irrelevant information as well as data measurement bias. Univariate FAVAR models are found to compete as well as or better than multivariate models in the case of inflation, suggesting that the direct inclusion of output does not provide additional information. For output models, however, multivariate FAVAR models perform better relative to their univariate counterparts indicating that the direct inclusion of inflation in the FAVAR model adds value. Simple forecast combinations were found to stably reduce the forecast error of benchmark model across the forecast horizon considered. These gains in forecasting accuracy were however relatively small when compared to competing models.

Specific asset prices have been found to produce superior forecasts of inflation and real GDP growth. In the case of inflation, these have included real and nominal exchange rates, gold prices, money supply indicators (M0 and M1) and the JSE stock price index. Individual predictors of real GDP growth were identified to be capacity utilisation, inflation, government bond yields (short and long) and short-term Treasury bill rates. Real house prices were also observed to be important indicators of real economic activity in the short to medium term. In some cases individual ARDL models were found to produce superior forecasts than the optimal models identified.

\section{References}

Ahmadi, P.A. \& Ritschl, A. 2007. Monetary policy during the great depression: a Bayesian FAVAR approach. In: Annual Congress of the European Economic Association and European Meeting of the Econometric Society: Budapest, Hungary, 27-31 August 2007.

Aiolfi, M. \& Timmermann, A. 2006. Persistence in forecasting performance and conditional combination strategies. Journal of Econometrics, 135(1-2):31-53.

Alessi, L., Barigozzi, M. \& Capasso, M. 2010. Improved penalization for determining the number of factors in approximate factor models. Statistics \& Probability Letters 80: 1806-1813.

Aron, J. \& Muellbauer, J. 2000. Inflation and output forecasting for South Africa: Monetary transmission implications. CSAE Working Paper Series 2000-23, Centre for the Study of African Economies: University of Oxford, Oxford.

Aron, J. \& Muellbauer, J. 2001. Interest rate effects on output: evidence from a GDP forecasting model for South Africa. CSAE Working Paper Series 2002-04, Centre for the Study of African Economies: University of Oxford, Oxford.

Bai, J. \& Ng, S. 2002. Determining the number of factors in approximate factor models. Econometrica, 70(1):191-221.

Banbura, M., Giannone, D. \& Reichlin, L. 2010. Large Bayesian vector auto regressions. Journal of Applied Econometrics, 25(1):71-92.

Bates, J.M. \& Granger, C.W. 1969. The combination of forecasts. Operations Research Qaurterly, 20:451468.

Bernanke, B.S. \& Blinder, A.S. 1992. The federal funds rate and the channels of monetary 
transmission. The American Economic Review, 82(4): 901-921.

Bernanke, B.S., Boivin, J. \& Eliasz, P. 2005. Measuring the effects of monetary policy: a factoraugmented vector autoregressive (FAVAR) approach. The Quarterly Journal of Economics, 120:387-422.

Bhardwaj, G. \& Swanson, N. R. 2006. An empirical investigation of the usefulness of ARFIMA models for predicting macroeconomic and financial time series. Journal of Econometrics, 131(1-2): 539578 .

Chan, Y.L., Stock, J.H. \& Watson, M.W. 1999. A dynamic factor model framework for forecast combination. Spanish Economic Review, 1(2):91-121.

Clark, T.E. \& McCracken, M.W. 2001. Test of equal forecast accuracy and forecast encompassing for nested models. Journal of Econometrics, 105(1):85-110.

Clemen, R.T. \& Winkler, R.L. 1986. Combining economic forecasts. Journal of Business and Economic Statistics, 4(1):39-46.

Clements, M.P. \& Hendry, D.F. 1996. Intercept corrections and structural change. Journal of Applied Econometrics, 11(5):475-494.

Clements, M.P. \& Hendry, D.F. 1998a. Forecasting economic processes. International Journal of Forecasting, 14(1):111-131.

Clements, M.P. \& Hendry, D.F. 1998b. Forecasting economic time series. Cambridge University

Press: Cambridge, UK.

Cook, S. 2004. Finite-sample properties of the GLS-based Dickey-Fuller test in the presence of breaks in innovation variance. Austrian Journal of Statistics, 33(3):305-314.

Das, S., Gupta, R. \& Kabundi, A. 2010. The blessings of dimensionality in forecasting real house price growth in the nine census divisions of the US. Journal of Housing Research, 19(1):89-109.

Doan, T.A., Litterman, R.B. \& Sims, C.A. 1984. Forecasting and conditional projections using realistic prior distributions. Econometric Reviews, 3(1):1-100.

Elliot, G., Rothenberg, T.J. \& Stock, J.H. 1996. Efficient tests for an Autoregressive unit root. Econometrica, 64(4):813-836.

Espinoza, R., Fornari, F. \& Lombardi, M.J. 2009. The role of financial variables in predicting economic activity. European Central Bank Working Paper Series, No. 1108.

Fedderke, J.W. \& Schaling, E. 2005. Modelling inflation in South Africa:a multivariate cointegration analysis. South African Journal of Economics, 73(1):79-92.

Forni, M., Hallin, M., Lippi, M. \& Reichlin, L. 2003. Do financial variables help forecasting inflation and real activity in the euro area? Journal of Monetary Economics, 50(6):1243-1255.

Forni, F. \& Lemke, W. 2010. Predicting recession probabilities with financial variables over multiple 
horizons. Working Paper Series, No.1255, European Central Bank.

Girouard, N. \& Blöndal, S. 2001. House prices and economic activity. OECD Economics Department Working Papers, No. 279, OECD Publishing.

Gupta, R. \& Kabundi, A. 2010. Forecasting macroeconomic variables in a small open economy: a comparison between small- and large-scale models. Journal of Forecasting, 29(1-2: Special Issue: Advances in Business Cycle Analysis and Forecasting): Pretoria: Department of Economics, University of Pretoria.

Gupta, R. \& Kabundi, A. Forecasting macroeconomic variables using large datasets: Dynamic factor model versus large-scale BVARs. Forthcoming in Indian Economic Review.

Gupta, R., Kabundi, A., Miller, S.M. \& Uwilingiye, J. 2011. Using large data sets to forecast sectoral employment. Working Papers, no. 2011-02, Connecticut: Department of Economics, University of Connecticut.

Gupta, R., Kabundi, A. \& Miller, S.M. Using large data sets to forecast house prices: a case study of twenty US states. Forthcoming in Journal of Housing Research.

Harvey, D.I., Leybourne, S.J. \& Newbold, P. 1998. Tests for forecast encompassing. Journal of Business and Economic Statictics, 16(2):254-259.

Hodrick, R.J. \& Prescott, E.C. 1997. Postwar U.S. business cycles: an empirical investigation. Journal of Money, Credit and Banking, 29(1):1-16.

Inoue, A. \& Kilian, L. 2008. How useful is bagging in forecasting economic time series? A case study of U.S. CPI inflation. Journal of the American Statistical Association, (103):511-522.

Kaseeram, I., Nichola, T. \& Mainardi, S. 2004. South African inflationary dynamics and the passthrough effects from depreciation to unit labour costs. South African Journal of Economics, 72(1):85-107.

Leamer, E.E. 2007. Housing is the business cycle. Working Paper 13428, National Bureau of Economic Research: Cambridge, MA.

Litterman, R. B. 1981. A Bayesian procedure for forecasting with Vector Autoregressions. Working Paper, Federal Reserve Bank of Minneapolis.

Litterman, R.B. 1986. Forecasting with Bayesian Vector Autoregressions: Five years of experience. Journal of Business and Economic Statistics, 4(1):25-38.

McCallum, B. 1983. A reconsideration of Sim's evidence concerning monetarism. Economic Letters, Elsevier 13(2-3):161-171.

Moolman, E. 2002. The term structure as a predictor of recessions. Studies in Economics and Econometrics, 26(3):43-52.

Nel, H. 1996. The term structure of interest rates and economic activity in South Africa. South African Journal of Economics, 64(3):161-174. 
Newey, W.K. \& West, K.D. 1987. A simple, positive semi-definite, heteroskedasticity and autocorrelation consistent covariance matrix. Econometrica, 55(3), 703-708.

Rapach, D.E. \& Strauss, J.K. 2005. Forecasting employment growth in Missouri with many potentially relevant predictors: an analysis of forecast coombining methods. Federal Reserve Bank of St. Louis Regional Economic Development, 1(1):97-112.

Rapach, D.E. \& Strauss, J.K. 2008. Forecasting U.S. employment growth using forecast combining methods. Journal of Forecasting, 27(1):75-93.

Rapach, D.E. \& Strauss, J.K. 2010. Bagging or combining (or both)?An analysis based on forecasting U.S. employment growth. Econometric Reviews, 29(5-6):511-533.

Rapach, D.E. \& Weber, C.E. 2004. Financial variables and the simulated out-of-sample forecastability of U.S. output growth since 1985: an encompassing approach. Economic Inquiry, 42(4):717-738.

Sims, C.A. 1980. Macroeconomics and Reality. Econometrica, 48(1):1-48.

Spencer, D.E. 1993. Developing a Bayesian vector autoregression model. International Journal of Forecasting, 9:407-421.

Stock, J.H. \& Watson, M.W. 1999. Forecasting inflation. NBER, Working Paper No. 7023.

Stock, J.H. \& Watson, M.W. 2001. Vector Autoregressions. Journal of Economic Perspectives, 15(4):101115.

Stock, J.H. \& Watson, M.W. 2002. Macroeconomics forecasting using diffusion indexes. Journal of Business and Economic Statictics, 20(2):157-162.

Stock, J.H. \& Watson, M.W. 2003. Forecasting output and inflation: the role of asset prices. Journal of Economic Literature, 41(3):788-829.

Stock, J.H. \& Watson, M.W. 2004. Combination forecasts of output growth in a seven country data set. Journal of Forecasting, 23(6):405-430.

Todd, R.M. 1984. Improving economic forecasting with Bayesian vector autoregression. Quarterly Review, Federal Reserve Bank of Minneapolis, Fall:18-29.

World Bank (www.worldbank.org).

Yang, Y. 2004. Combining forecasting procedures: some theoretical results. Econometric Theory, 20(1):176-222.

Zaher, F. 2007. Evaluating factor forecasts for the UK: the role of asset prices. International Journal of Forecasting, 23(4):679-693. 


\section{Appendix A - Data Sources by Variable}

\begin{tabular}{|c|c|c|c|}
\hline $\begin{array}{r}\text { Variable } \\
\text { Asset Prices }\end{array}$ & Data Source / Calculation & Data Sample Periods & Transformation \\
\hline EXRATE & IMF International Financial Statistics & 1980:Q1 - 2010:Q3, m & $\begin{array}{l}\text { Seasonally Adjusted, logged and } \\
\text { first differenced }\end{array}$ \\
\hline DIVPR & Global Financial Database & 1980:Q1 - 2010:Q4, m & Level \\
\hline GOLD & Bloomberg & 1980:Q1 - 2010:Q4, d & $\begin{array}{l}\text { Seasonally Adjusted, logged and } \\
\text { first differenced }\end{array}$ \\
\hline HOUSE & ABSA & 1966:Q1 - 2010:Q3, m & $\begin{array}{l}\text { Logged and first differenced } \\
\text { (already seasonally adjusted) }\end{array}$ \\
\hline RBNDL & South African Reserve Bank & 1980:Q1 - 2010:Q4, m & Level and first differenced \\
\hline RBNDS & South African Reserve Bank & 1980:Q1 - 2010:Q4, m & Level and first differenced \\
\hline REPO & South African Reserve Bank & 1980:Q1 - 2010:Q4, d & Level and first differenced \\
\hline REXRATE & IMF International Financial Statistics & 1980:Q1 - 2010:Q3, m & $\begin{array}{l}\text { Seasonally Adjusted, logged and } \\
\text { first differenced }\end{array}$ \\
\hline RGOLD & $\begin{array}{l}\text { Real gold price (per ounce, R): } \\
\text { GOLD/CPI*100 }\end{array}$ & 1980:Q1 - 2010:Q3, d & $\begin{array}{l}\text { Seasonally Adjusted, logged and } \\
\text { first differenced }\end{array}$ \\
\hline RHOUSE & $\begin{array}{l}\text { Real ABSA House price index: } \\
\text { HOUSE/CPI*100 }\end{array}$ & 1966:Q1 - 2010:Q3, m & $\begin{array}{l}\text { Logged and first differenced } \\
\text { (already seasonally adjusted) }\end{array}$ \\
\hline RRBNDL & $\begin{array}{l}\text { Government bond yield, } 10 \text { years and over } \\
(\%) \text { : RBNDL-inflation }\end{array}$ & 1980:Q1 - 2010:Q4, m & Level and first differenced \\
\hline RRBNDS & $\begin{array}{l}\text { Government bond yield, } 0-3 \text { years }(\%) \text { : } \\
\text { RBNDS-inflation }\end{array}$ & 1980:Q1 - 2010:Q4, m & Level and first differenced \\
\hline RREPO & Real policy rate: REPO-inflation & 1980:Q1 - 2010:Q4, d & Level and first differenced \\
\hline RRTBILL & Real treasury bill rate (\%): RTBILL-inflation & 1980:Q1 - 2010:Q4, q & Level and first differenced \\
\hline RSILVER & $\begin{array}{l}\text { Real silver price (per ounce, } \mathrm{R}) \text { : } \\
\text { SILVER/CPI*100 }\end{array}$ & 1980:Q1 - 2010:Q4, d & $\begin{array}{l}\text { Seasonally Adjusted, logged and } \\
\text { first differenced }\end{array}$ \\
\hline RSPREAD & Term spread: RBNDL-RTBILL & 1980:Q1 - 2010:Q4, q & Level \\
\hline RSTOCKP & $\begin{array}{l}\text { Real JSE stock price index: } \\
\text { STOCKP/CPI*100 }\end{array}$ & 1980:Q1 - 2010:Q4, q & $\begin{array}{l}\text { Seasonally Adjusted, logged and } \\
\text { first differenced }\end{array}$ \\
\hline RTBILL & IMF International Financial Statistics & 1980:Q1 - 2010:Q4, q & Level and first differenced \\
\hline SILVER & Bloomberg & 1980:Q1 - 2010:Q4, d & $\begin{array}{l}\text { Seasonally Adjusted, logged and } \\
\text { first differenced }\end{array}$ \\
\hline STOCKP & South African Reserve Bank & 1980:Q1 - 2010:Q4, q & $\begin{array}{l}\text { Seasonally Adjusted, logged and } \\
\text { first differenced }\end{array}$ \\
\hline
\end{tabular}




\section{Appendix A - Data Sources by Variable (continued)}

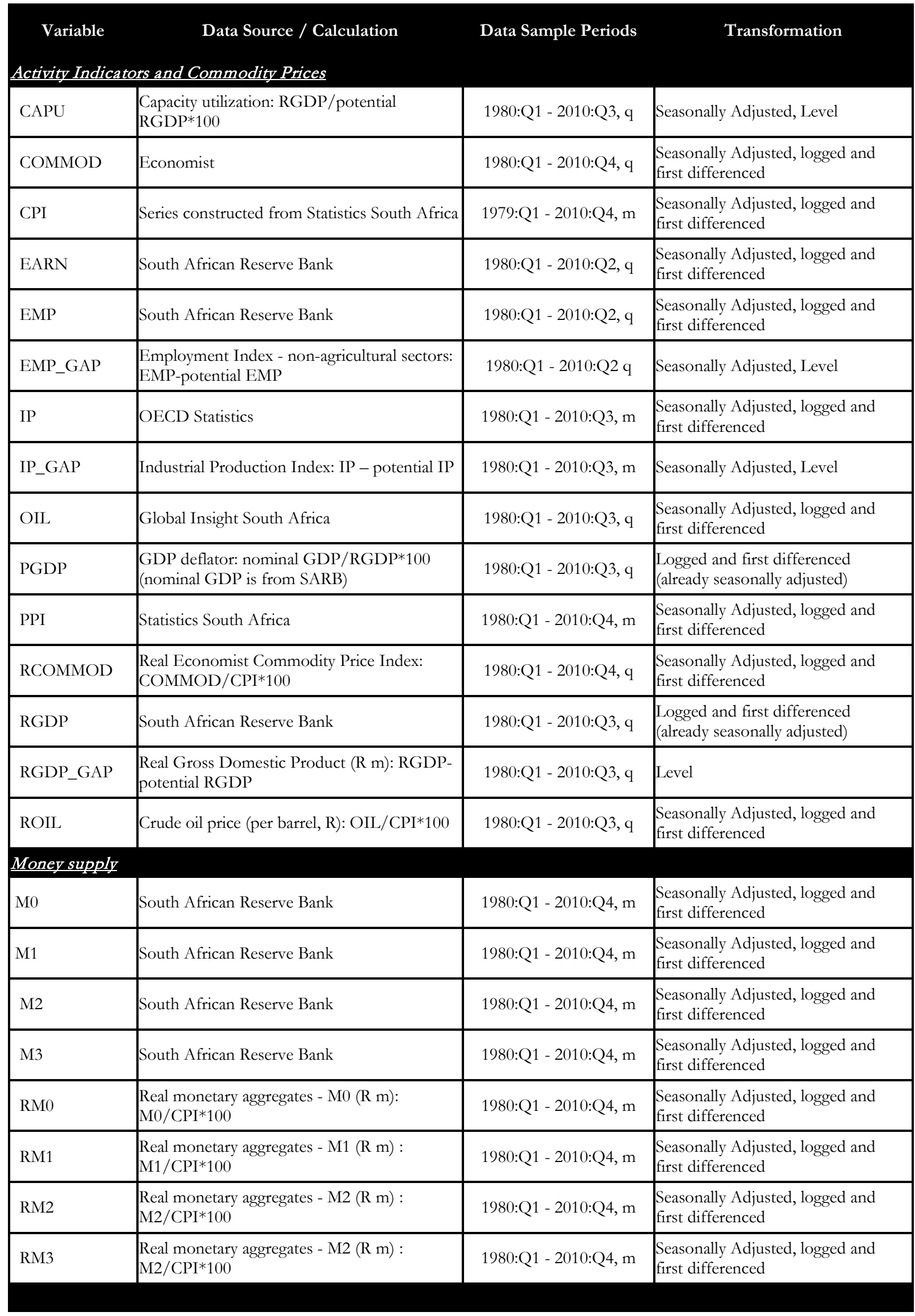


Appendix B - Data Summary Statistics

\begin{tabular}{|c|c|c|c|c|c|c|}
\hline $\begin{array}{r}\text { Variable } \\
\text { Asset Prices }\end{array}$ & $\begin{array}{l}\text { Number of } \\
\text { Observations }\end{array}$ & Mean & $\begin{array}{l}\text { Standard } \\
\text { Deviation }\end{array}$ & Minimum & Maximum & Skewness \\
\hline DIVPR & 121 & 3.256 & 0.896 & 1.863 & 6.120 & 0.791 \\
\hline DLEXRATE & 121 & -0.017 & 0.057 & -0.239 & 0.136 & -0.668 \\
\hline DLGOLD & 121 & 0.023 & 0.067 & -0.138 & 0.235 & 0.266 \\
\hline DLHOUSE & 121 & 0.029 & 0.025 & -0.029 & 0.091 & 0.058 \\
\hline DLREXRATE & 121 & -0.003 & 0.056 & -0.208 & 0.189 & -0.269 \\
\hline DLRGOLD & 121 & 0.000 & 0.069 & -0.174 & 0.208 & 0.132 \\
\hline DLRHOUSE & 121 & 0.006 & 0.031 & -0.072 & 0.083 & -0.080 \\
\hline DLRSILVER & 121 & -0.010 & 0.125 & -0.807 & 0.260 & -2.027 \\
\hline DLRSTOCKP & 121 & -0.002 & 0.105 & -0.552 & 0.160 & -1.779 \\
\hline DLSILVER & 121 & 0.013 & 0.122 & -0.771 & 0.266 & -2.070 \\
\hline DLSTOCKP & 121 & 0.021 & 0.104 & -0.532 & 0.192 & -1.825 \\
\hline DRBNDL & 121 & -0.003 & 0.797 & -2.000 & 3.300 & 0.619 \\
\hline DRBNDS & 121 & 0.018 & 1.185 & -4.300 & 4.700 & -0.082 \\
\hline DREPO & 121 & 0.015 & 1.211 & -3.600 & 5.100 & 0.316 \\
\hline DRRBNDL & 121 & 0.078 & 1.565 & -3.690 & 4.870 & 0.264 \\
\hline DRRBNDS & 121 & 0.099 & 1.688 & -4.826 & 4.270 & -0.118 \\
\hline DRREPO & 121 & 0.096 & 1.533 & -4.120 & 4.000 & -0.094 \\
\hline DRRTBILL & 121 & 0.101 & 1.658 & -3.790 & 3.511 & -0.036 \\
\hline DRTBILL & 121 & 0.020 & 1.289 & -3.900 & 6.100 & 0.523 \\
\hline RSPREAD & 121 & 1.108 & 2.757 & -4.800 & 6.800 & 0.177 \\
\hline \multicolumn{7}{|c|}{ Activity Indicators and Commodity Prices } \\
\hline CAPU & 121 & 100.145 & 1.847 & 95.995 & 104.388 & 0.123 \\
\hline DLCOMMOD & 121 & 0.004 & 0.061 & -0.378 & 0.145 & -1.740 \\
\hline DLCPI & 121 & 0.023 & 0.013 & -0.020 & 0.061 & -0.138 \\
\hline DLEARN & 121 & 0.004 & 0.019 & -0.038 & 0.077 & 0.940 \\
\hline DLEMP & 121 & 0.004 & 0.032 & -0.024 & 0.324 & 8.332 \\
\hline DLIP & 121 & 0.003 & 0.023 & -0.070 & 0.073 & -0.220 \\
\hline DLOIL & 121 & 0.023 & 0.136 & -0.641 & 0.361 & -1.234 \\
\hline DLPGDP & 121 & 0.025 & 0.015 & -0.015 & 0.063 & 0.213 \\
\hline DLPPI & 121 & 0.022 & 0.014 & -0.022 & 0.063 & -0.092 \\
\hline DLRCOMMOD & 121 & -0.019 & 0.063 & -0.389 & 0.132 & -1.325 \\
\hline DLRGDP & 121 & 0.006 & 0.009 & -0.021 & 0.025 & -0.637 \\
\hline DLROIL & 121 & 0.000 & 0.138 & -0.701 & 0.329 & -1.339 \\
\hline EMP_GAP & 121 & 0.021 & 4.896 & -18.219 & 17.108 & -0.527 \\
\hline$\overline{\text { IP_GAP }}$ & 121 & 0.045 & 3.158 & -9.805 & 10.833 & 0.250 \\
\hline RGDP_GAP & 121 & 439.410 & 20476.850 & -42056.660 & 56140.920 & 0.340 \\
\hline \multicolumn{7}{|l|}{ Money supply } \\
\hline DLM0 & 121 & 0.036 & 0.023 & -0.015 & 0.150 & 1.263 \\
\hline DLM1 & 121 & 0.040 & 0.037 & -0.128 & 0.133 & -0.677 \\
\hline DLM2 & 121 & 0.037 & 0.022 & -0.020 & 0.086 & -0.044 \\
\hline DLM3 & 121 & 0.035 & 0.017 & -0.006 & 0.076 & 0.060 \\
\hline DLRM0 & 121 & 0.013 & 0.025 & -0.037 & 0.132 & 0.926 \\
\hline DLRM1 & 121 & 0.017 & 0.039 & -0.167 & 0.113 & -0.879 \\
\hline DLRM2 & 121 & 0.014 & 0.024 & -0.043 & 0.059 & -0.319 \\
\hline DLRM3 & 121 & 0.012 & 0.021 & -0.038 & 0.064 & -0.075 \\
\hline
\end{tabular}

